Proceedings of the Edinburgh Mathematical Society (2008) 51, 229-263 (C)

DOI:10.1017/S0013091505000337 Printed in the United Kingdom

\title{
THE OPERATOR SHIFT SPACE
}

\author{
TIMUR OIKHBERG \\ Department of Mathematics, University of California, Irvine, \\ Irvine, CA 92697, USA (toikhber@math.uci.edu)
}

(Received 14 March 2005)

\begin{abstract}
We construct and examine an operator space $X$, isometric to $\ell_{2}$, such that every completely bounded map from its subspace $Y$ into $X$ is a compact perturbation of a linear combination of multiples of a shift of given multiplicity and their adjoints. Moreover, every completely bounded map on $X$ is a Hilbert-Schmidt perturbation of such a linear combination.
\end{abstract}

Keywords: operator spaces; completely bounded maps; completely indecomposable spaces; shift operator

2000 Mathematics subject classification: Primary 46L07; 46L10; 47L25

\section{Introduction and the main result}

In [8], Gowers and Maurey constructed a Banach space $X_{s}$ such that any $T \in B\left(X_{s}\right)$ is a strictly singular perturbation of a Toeplitz operator. In this paper, we present a similar construction in the operator space case. In fact, the space $X$ constructed below has certain additional properties. For instance, a linear operator on $X$ is completely bounded if and only if it is a Hilbert-Schmidt perturbation of a Toeplitz operator with coefficients in a certain weighted $\ell_{1}$ space.

Throughout the paper, we freely use standard operator space results and terminology. The reader is referred to $[\mathbf{6}, \mathbf{2 3}]$ for more information. We work mainly with operator spaces isometric to $\ell_{2}$. The shift operator $S \in B\left(\ell_{2}\right)$ is defined by setting $S \delta_{i}=\delta_{i+1}$ for $i \in \mathbb{N}$ (throughout the paper, $\left(\delta_{i}\right)_{i \in \mathbb{N}}$ denotes the canonical basis in $\ell_{2}$ ). For the sake of brevity, we shall use the notation $S^{-1}$ for $S^{*}$ (that is, $S^{-1} \delta_{1}=0, S \delta_{i}=\delta_{i-1}$ for $i \geqslant 2$ ).

Some additional notation will be used in the paper. We use $\mathcal{S}_{p}, 1 \leqslant p \leqslant \infty$, to denote a Schatten class of operators, while $\|\cdot\|_{p}$ is the corresponding norm. Throughout the paper, $C>1$ and $m \in \mathbb{N}$ are fixed. $\ell_{1}\left(C^{|j|}, \mathbb{Z}\right)$ denotes the weighted $\ell_{1}$ space of all sequences $a=\left(a_{j}\right)_{j \in \mathbb{Z}}$, equipped with the norm $\|a\|_{w}=\sum_{j \in \mathbb{Z}} C^{|j|}\left|a_{j}\right|$. We define the Toeplitz operator $\tilde{T}_{a}$ as

$$
\tilde{T}_{a}=\sum_{j \geqslant 0} a_{j} S^{m j}+\sum_{j<0} a_{j} S^{* m j} \in B\left(\ell_{2}\right) .
$$

Our main result is the following theorem. 
Theorem 1.1. For every $m \in \mathbb{N}$ and $C>1$ there exists an operator space $X=$ $X(C, m)$, isometric to $\ell_{2}$, such that the following conditions hold.

(i) (a) Any operator of the form $\tilde{T}_{a}+W \in B(X)$, with $a \in \ell_{1}\left(C^{|j|}, \mathbb{Z}\right)$ and $W \in \mathcal{S}_{2}$, is completely bounded, with $\left\|\tilde{T}_{a}+W\right\|_{\mathrm{cb}} \leqslant\|a\|_{w}+\|W\|_{2}$.

(b) Conversely, every $T \in \mathrm{CB}(X)$ can be written as $T=\tilde{T}_{a}+W$, with $a \in$ $\ell_{1}\left(C^{|j|}, \mathbb{Z}\right), W \in \mathcal{S}_{2},\|W\|_{2} \leqslant \gamma\|T\|_{\mathrm{cb}}$ and $\|a\|_{w} \leqslant 4\|T\|_{\mathrm{cb}}$. Here, the constant $\gamma$ depends only on $C$ and $m$.

(ii) If $Y$ is an infinite-dimensional subspace of $X$, and $T \in \mathrm{CB}(Y, X)$, then $T-\tilde{T}_{a}$ is compact for some $a \in \ell_{1}\left(C^{|j|}, \mathbb{Z}\right)$ satisfying $\|a\|_{w} \leqslant 4\|T\|_{\mathrm{cb}}$.

(iii) Any infinite-dimensional subspace of $X$ fails the operator approximation property (OAP).

(iv) $K_{0}(\mathrm{CB}(X))=\mathbb{Z} \oplus \mathbb{Z} / m \mathbb{Z}$ and $K_{1}(\mathrm{CB}(X))=\{0\}$.

It is instructive to compare this statement with the results of Gowers and Maurey [8, 13, 14]. For any $m$, they construct a Banach space $X_{s}=X_{s}(m)$, equipped with a basis $\left(e_{i}\right)$, such that

(1) the left and right shifts with respect to this basis, denoted by $L$ and $R$, respectively, are contractive, and

(2) for any $T \in B\left(Y, X_{s}\right)$ ( $Y$ is a subspace of $X_{s}$ ), there exists a sequence $\left(a_{j}\right)_{j \in \mathbb{Z}} \in$ $\ell_{1}(\mathbb{Z})$ such that

$$
\left\|T-\left(\sum_{j \geqslant 0} a_{j} R^{m j}+\sum_{j<0} a_{j} L^{-m j}\right)\right\|=0 .
$$

Here, the semi-norm $\|W W\|$ is described in terms the action of an operator $W$ on block bases in $X$, in a rather involved way. It is known that $W$ is compact $\Rightarrow\|W\|=0 \Rightarrow W$ is strictly singular. However, we are not aware of any explicit descriptions of $B\left(X_{s}\right)$. In this way, Theorem 1.1 (ii) is similar to known results, and (i) is an improvement.

As noted above, the shift space of Gowers and Maurey has a basis; hence, it has the approximation property. We do not know if this property is inherited by all of its infinitedimensional subspaces. Thus, (iii) underscores the difference between the classical and non-commutative cases.

Finally, Theorem 1.1 (iv) is similar to an exercise following [13, Theorem 12.1], stating that $K_{0}\left(\mathrm{CB}\left(X_{s}\right)\right)=\mathbb{Z} \oplus \mathbb{Z} / m \mathbb{Z}$ and $K_{1}\left(\mathrm{CB}\left(X_{s}\right)\right)=\{0\}$.

Motivation for this paper comes in part from the work on representations of Banach algebras as spaces of (completely) bounded maps. It was proved by Berkson and Porta $[\mathbf{1}]$ that $B\left(\ell_{p}\right)$ (for $p \in[1,2) \cup(2, \infty]$ ) has no non-trivial representations on $B\left(\ell_{2}\right)$. Following the work of Størmer [26], Neufang, Popa, Ruan, Smith and Spronk $[\mathbf{1 6}, \mathbf{2 4}, \mathbf{2 5}]$ recently established that certain group algebras can be represented isometrically as subalgebras of $\mathrm{CB}(B(H))$, where $H$ is a suitable Hilbert space. In fact, in many cases the group 
algebra can be identified with the algebra of module maps, satisfying certain continuity properties.

We are interested in a similar problem: suppose that $\mathcal{A}$ is a unital Banach algebra and $\pi: \mathcal{A} \rightarrow B(H)$ is a unital representation. Can $H$ be equipped with an operator space structure $X$ such that $\mathrm{CB}(X)$ consists of 'small' (usually, Hilbert-Schmidt) perturbations of elements of $\pi(\mathcal{A})$ ? The study of this question begun in [21], where we gave a positive answer when $\mathcal{A}$ is a hyperfinite von Neumann subalgebra of $B\left(\ell_{2}\right)$, and $\pi$ is the identity representation. In [19], we proved that the answer is positive when $H=\ell_{2} \otimes_{2} \ell_{2}, \mathcal{A}$ is a dual Banach algebra, and $\pi=\rho \otimes I_{\ell_{2}}$, where $\rho: \mathcal{A} \rightarrow B\left(\ell_{2}\right)$ is a contractive, unital, weakly* continuous representation. A positive answer is given in $[\mathbf{2 0}]$ when $\mathcal{A}$ is the bidual of the James quasi-reflexive space (viewed as a commutative Banach algebra).

In this paper, we consider the weighted convolution algebras $\ell\left(C^{|j|}, \Gamma\right)$, where the semigroup $\Gamma$ is either $\mathbb{Z}$ or $\mathbb{Z}^{+}=\mathbb{N} \cup\{0\}$ and $C>1$. In other words, we work with the set of (bi-)infinite sequences

$$
\left(a_{j}\right)_{j \in \Gamma}=\sum_{j \in \Gamma} a_{j} \delta_{j}
$$

equipped with the norm $\left\|\left(a_{j}\right)_{j \in \Gamma}\right\|_{w}=\sum_{j \in \Gamma} C^{|j|}\left|a_{j}\right|$, and with the multiplication $\delta_{s}$ * $\delta_{t}=\delta_{s+t}$ for $s, t \in \Gamma$ (see, for example, $[\mathbf{4}, \S 4.6]$ for properties of such algebras). The map $\psi: \ell_{1}\left(C^{|j|}, \mathbb{Z}\right) \rightarrow B\left(\ell_{2}\right): \delta_{j} \mapsto S^{m j}$ is not a representation, but 'close to', and $\mathrm{CB}(X)$ consists of Hilbert-Schmidt perturbations of elements of $\psi\left(\ell_{1}\left(C^{|j|}, \mathbb{Z}\right)\right)$. In $\S 8$ we consider some faithful representations $\pi: \ell\left(C^{|j|}, \Gamma\right) \rightarrow B(H)$, where $H$ is $\ell_{2}$ or $\ell_{2}(\mathbb{Z})$. By modifying the reasoning of $\S \S 2-7$, we equip $H$ with an operator space structure $X(\pi)$ in such a way that $\pi: \ell\left(C^{|j|}, \Gamma\right) \rightarrow \mathrm{CB}(X(\pi))$ becomes a complete isometry and, moreover, $\mathrm{CB}(X(\pi))=\pi\left(\ell\left(C^{|j|}, \Gamma\right)\right)+\mathcal{S}_{2}$. In Theorem 8.1, we list some properties of the spaces $X(\pi)$. These spaces do not seem to be as interesting as the space $X=X(C, m)$, and hence we mention them only briefly.

The rest of the paper is structured as follows. In $\S 2$, we construct the space $X=$ $X(C, m)$ (henceforth, this notation will be reserved for the operator space constructed there), establish the groundwork for the rest of the paper and prove Theorem 1.1 (i) (a). The key part is $\S 3$, in which we show that for every $T \in \mathrm{CB}(Y, X)$ (here $Y$ is a subspace of $X)$ there exists $a \in \ell_{1}\left(C^{|j|}, \mathbb{Z}\right)$ such that $T-\tilde{T}_{a}$ is compact. Based on this, we establish Theorem 1.1 (ii). In addition, we show that no coordinate subspace of $X$ has non-trivial completely bounded (c.b.) projections (Theorem 3.1), and that a subspace $Y \hookrightarrow X$ is completely isomorphic to $X$ if and only if $\operatorname{dim} X / Y$ is finite, and divisible by $m$ (Theorem 3.2). Consequently, the space $X(C, 1)$ is completely prime (Corollary 3.3).

In $\S 4$ we show that, for certain $Z \hookrightarrow X$, for any $T \in \mathrm{CB}(Z, X)$ there exists $a \in$ $\ell_{1}\left(C^{|j|}, \mathbb{Z}\right)$ such that $T-\tilde{T}_{a}$ is Hilbert-Schmidt. In particular, this happens when $Z=X$, thus completing the proof of Theorem 1.1 (i). Moreover, any infinite-dimensional $Y \hookrightarrow X$ contains an infinite-dimensional $Z$ with the above property (Theorem 4.1).

Sections 5 and 6 are devoted to proving that infinite-dimensional subspaces of $X$ fail the OAP, and to computing the $K$-groups of $\mathrm{CB}(X)$ (parts (iii) and (iv) of Theorem 1.1, respectively). In $\S 7$ we establish some additional properties of the space $X=X(C, m)$, and its subspaces. We show that, for general $Y \hookrightarrow X$, compact operators in $\mathrm{CB}(Y, X)$ 
may fail to be Hilbert-Schmidt, or have certain non-commutative properties analogous to compactness (compare with Theorem 1.1(i), stating that any compact $T \in \mathrm{CB}(X)$ is Hilbert-Schmidt). Finally, in $\S 8$ we present a version of our construction, involving representations of weighted convolution algebras.

\section{The construction of $X(C, m)$}

In this section, we construct the space $X=X(C, m)$ as in Theorem 1.1. Recall that, by $[\mathbf{2 1}]$, there exists a family $\left(E_{i}\right)_{i=1}^{\infty}$ of finite-dimensional operator spaces such that

(i) $E_{i}$ is isometric to $\ell_{2}^{n_{i}}$ for some $n_{i} \in \mathbb{N}$, and $\left\{i \mid n_{i}=j\right\}$ is infinite for any $j \in \mathbb{N}$,

(ii) for any operator $u: E_{i}^{*} \rightarrow E_{j}$, we have $\|u\|_{1} /\left(4+2^{-i}\right) \leqslant\|u\|_{\mathrm{cb}} \leqslant\|u\|_{1}$ if $i=j$, $\|u\|_{\mathrm{cb}}=\|u\|_{2}$ if $i \neq j$.

Denote by $\mathcal{K}$ the space of compact operators on $\ell_{2}$. Find a sequence of operators $u_{i}: \ell_{2} \rightarrow \ell_{2}^{n_{i}}$ such that $\left\|u_{i}\right\|_{2}=1$ and, for any $\varepsilon>0, n \in \mathbb{N}$, and for any $u: \ell_{2} \rightarrow \ell_{2}^{n}$ satisfying $\|u\|_{2}=1$, there exists $i \in \mathbb{N}$, for which $n_{i}=n$ and $\left\|u_{i}-u\right\|_{1}<\varepsilon$. On the Banach space level, we identify the range of $u_{i}$ with $E_{i}$ described above.

We define the operator space $X=X(C, m)$ as follows: for $x \in \ell_{2} \otimes \mathcal{K}$, let

$$
\left.\begin{array}{rl}
\mathcal{U} & =\left\{C^{-(p+q)} S^{m p} S^{* m q} \mid p, q \in \mathbb{N} \cup\{0\}\right\}, \\
\|x\|_{X \otimes \mathcal{K}} & =\sup \left\{\left\|\left(u_{i} U \otimes I_{\mathcal{K}}\right) x\right\|_{E_{i} \otimes \mathcal{K}} \mid i \in \mathbb{N}, U \in \mathcal{U}\right\} .
\end{array}\right\}
$$

Clearly, $X$ is an operator space (Ruan's axioms are satisfied), $X$ is isometric to $\ell_{2}$ as a Banach space and $\left\|S^{m}\right\|_{\mathrm{cb}},\left\|S^{* m}\right\|_{\mathrm{cb}} \leqslant C\left(S^{*} S=I\right.$, and hence a product of any number of copies of $S$ and $S^{*}$ in an arbitrary order can be written as $S^{p} S^{* q}$, where $p$ and $q$ are non-negative integers). Moreover, 2 -summing operators with the range in $X$ (and with arbitrary domain) are completely bounded (see the following lemma).

Lemma 2.1. If $Y$ is an operator space, and $T: Y \rightarrow X$ is a 2-summing operator, then $\|T\|_{\mathrm{cb}} \leqslant \pi_{2}(T)$. If $Y$ is isometric to a Hilbert space, and $T: Y \rightarrow X$ is a Hilbert-Schmidt operator, then $\|T\|_{\mathrm{cb}} \leqslant\|T\|_{2}$.

Proof. By (2.1),

$$
\|T\|_{\mathrm{cb}}=\sup \left\{\left\|u_{i} U T\right\|_{\mathrm{cb}} \mid i \in \mathbb{N}, U \in \mathcal{U}\right\} \leqslant \sup \left\{\left\|u_{i} U T\right\|_{1} \mid i \in \mathbb{N}, U \in \mathcal{U}\right\} .
$$

By [9, Statement 4.5], $\left\|u_{i} U T\right\|_{1} \leqslant\left\|u_{i}\right\|_{2}\|U\| \pi_{2}(T) \leqslant \pi_{2}(T)$ for such $i$ and $U$. Thus, $\|T\|_{\mathrm{cb}} \leqslant \pi_{2}(T)$. If $Y$ is isometric to a Hilbert space, then $\pi_{2}(T)=\|T\|_{2}$ (see, for example, $[\mathbf{9}$, Statement 3.9]) and we are done.

Thus, Theorem 1.1 (i) (a) has been established. To deal with the rest of the theorem, we need to obtain lower estimates for c.b. norms of operators on $X$. The following lemma is one of our main tools. 
Lemma 2.2. Suppose that $Y$ is a subspace of $X$. Consider the operators $T: Y \rightarrow X$, $u: X \rightarrow \ell_{2}^{n}$ and $v: \ell_{2}^{n} \rightarrow Y$, such that $\|u\|_{2}=1=\|v\|$. Let

$$
c=\sup \left\{C^{-(p+q)}\left\|u S^{m p} S^{* m q} v\right\|_{1} \mid p, q \in \mathbb{N} \cup\{0\}\right\} .
$$

Then $\|T\|_{\mathrm{cb}} \geqslant\|u T v\|_{1} /(4 \max \{c, 1\})$.

Proof. Fix $\varepsilon>0$. By construction, there exist infinitely many $i \in \mathbb{N}$ such that $i>\varepsilon^{-1}$, $n_{i}=n$ and $\left\|u_{i}-u\right\|_{1}<\varepsilon$ (we identify $\ell_{2}^{n}$ with $E_{i}$ ). We view $u$ and $v$ as maps from $X$ to $E_{i}$ and from $E_{i}^{*}$ to $X$, respectively. By $(2.1)$,

$$
\|v\|_{\mathrm{cb}}=\sup \left\{C^{-(p+q)}\left\|u_{j} S^{m p} S^{* m q} v\right\|_{\mathrm{cb}} \mid j \in \mathbb{N}, p, q \in \mathbb{N} \cup\{0\}\right\} .
$$

If $i=j$, then

$$
C^{-(p+q)}\left\|u_{j} S^{m p} S^{* m q} v\right\|_{\mathrm{cb}} \leqslant C^{-(p+q)}\left(\left\|u S^{m p} S^{* m q} v\right\|_{1}+\left\|u-u_{i}\right\|_{1}\right) \leqslant c+\varepsilon
$$

for any $p, q \in \mathbb{N} \cup\{0\}$. If $j \neq i$,

$$
C^{-(p+q)}\left\|u_{j} S^{m p} S^{* m q} v\right\|_{\mathrm{cb}} \leqslant\left\|u_{j} S^{m p} S^{* m q} v\right\|_{2} \leqslant\left\|u_{j}\right\|_{2}\left\|S^{m p} S^{* m q}\right\|\|v\|=1 .
$$

Therefore, $\|v\|_{\mathrm{cb}} \leqslant \max \{c+\varepsilon, 1\}$.

By (2.1), $\left\|u_{i}\right\|_{\mathrm{cb}}=1$, and therefore $\|u\|_{\mathrm{cb}} \leqslant\left\|u_{i}\right\|_{\mathrm{cb}}+\left\|u-u_{i}\right\|_{1}=1+\varepsilon$. By the definition of $E_{i},\|w\|_{\mathrm{cb}} \geqslant\|w\|_{1} /(4+\varepsilon)$ for any $w \in \mathrm{CB}\left(E_{i}^{*}, E_{i}\right)$, and therefore

$$
\|T\|_{\mathrm{cb}} \geqslant \frac{\|u T v\|_{\mathrm{cb}}}{\|u\|_{\mathrm{cb}}\|v\|_{\mathrm{cb}}} \geqslant \frac{\|u T v\|_{1}}{(4+\varepsilon)(1+\varepsilon) \max \{c+\varepsilon, 1\}} .
$$

However, $\varepsilon$ can be chosen to be arbitrarily small.

\section{Extracting the Toeplitz part}

The main goal of this section is to prove part (ii) of Theorem 1.1, namely, that every element of $\mathrm{CB}(Y, X)(Y$ is a subspace of $X)$ is a compact perturbation of the restriction of a Toeplitz operator to $Y$. We also establish the following theorem.

Theorem 3.1. Suppose that $Y$ is a coordinate subspace of $X$ (that is, $Y=\operatorname{span}\left[\delta_{i} \mid\right.$ $i \in \mathcal{I}]$ for some $\mathcal{I} \subset \mathbb{N}$ ), and $P \in \mathrm{CB}(Y)$ is a projection. Then $P$ has finite-dimensional kernel or finite-dimensional range.

Theorem 3.2. Suppose that $X^{\prime}$ is a subspace of $X$ of finite codimension and $Y$ is a subspace of $X$. Then $X^{\prime}$ is completely isomorphic to $Y$ if and only if $\operatorname{dim} X / Y$ is finite, and $\operatorname{dim} X / X^{\prime}-\operatorname{dim} X / Y$ is divisible by $m$.

Recall that an operator space $Z$ is called completely prime if, for any projection $P \in$ $\mathrm{CB}(Z)$, either ran $P$ or ker $P$ is completely isomorphic to $Z$.

Corollary 3.3. The space $X(C, 1)$ is completely prime for any $C>1$. 
Proof. By Theorem 3.1, any projection $P \in \mathrm{CB}(X(C, 1))$ with infinite rank has finitedimensional kernel. By Theorem 3.2, $\operatorname{ran} P$ is completely isomorphic to $X(C, 1)$.

For the proofs, we need certain special orthonormal bases $\left(\xi_{i}\right)$, modelled on lacunary sequences, in the sense that $\left\langle S^{m j_{1}} \xi_{i_{1}}, S^{m j_{2}} \xi_{i_{2}}\right\rangle=0$ if $i_{1} \neq i_{2}$, and $\left|j_{1}\right|+\left|j_{2}\right|$ is sufficiently small. More precisely, let $\alpha=\frac{1}{2} \min \{1, \ln C\}$. For $i \in \mathbb{N}$, let $K_{i}=\lfloor i / \alpha\rfloor$. A sequence $\left(\xi_{i}\right)_{i \in \mathbb{N}}$ in $\ell_{2}$ is called shift lacunary if it is normalized, and

(i) for each $i \in \mathbb{N}, \operatorname{supp} \xi_{i}=\left\{r \in \mathbb{N} \mid\left\langle\xi_{i}, \delta_{r}\right\rangle \neq 0\right\} \subset\left[2 m\left(K_{i}+2\right), \infty\right)$,

(ii) there exists a sequence of mutually orthogonal subspaces $\left(F_{i}\right)_{i \in \mathbb{N}}$ of $\ell_{2}$, such that $\operatorname{dim} F_{i}=2 K_{i}+1$, and $S^{m j} \xi_{i} \in F_{i}$ whenever $|j| \leqslant K_{i}$.

Note that the above definition implies that $\left\langle\xi_{i}, \xi_{k}\right\rangle=0$ if $i \neq k$. More properties of shift lacunary sequences are mentioned below, in (3.1).

Proposition 3.4. Suppose that $\left(Y_{n}\right)$ is a sequence of infinite-dimensional subspaces of $\ell^{2}$. There then exists a shift lacunary sequence $\left(\xi_{n}\right)_{n \in \mathbb{N}}$, so that $\xi_{n} \in Y_{n}$ for any $n$.

Proof. Without loss of generality, we can assume that $\operatorname{supp} \xi \subset\left[2 m\left(K_{i}+2\right), \infty\right)$ whenever $\xi \in Y_{i}, i \in \mathbb{N}$.

We shall select a shift lacunary sequence $\left(\xi_{i}\right)$ inductively. Pick an arbitrary norm-1 $\xi_{1} \in Y_{1}$, and an arbitrary subspace $F_{1}$ of $\ell^{2}$, containing $S^{m j} \xi_{1}$ for $|j| \leqslant K_{1}$. Now suppose that $\xi_{1}, \ldots, \xi_{n-1}$ and $F_{1}, \ldots, F_{n-1}$, satisfying the definition above, have been chosen, and pick $\xi_{n}$ and $F_{n}$. Pick an arbitrary norm-1 vector

$$
\xi_{n} \in Y_{n} \cap\left(\bigcup_{\substack{|j| \leqslant K_{n}, 1 \leqslant k \leqslant n-1}} S^{m j} F_{k}\right)^{\perp} .
$$

Then $\operatorname{supp} \xi_{n} \subset\left[2 m\left(K_{n}+2\right), \infty\right)$, and $\left\langle S^{m j} \xi_{n}, \eta\right\rangle=\left\langle\xi_{n}, S^{-m j} \eta\right\rangle=0$ whenever $|j| \leqslant K_{n}$, and $\eta \in F_{k}$ for some $k \in\{1, \ldots, n-1\}$. To complete the construction, let $F_{n}$ be an arbitrary subspace of $\ell_{2}$ of dimension $2 K_{n}+1$, containing $S^{m j} \xi_{n}$ whenever $|j| \leqslant K_{n}$, and orthogonal to the spaces $F_{k}$ for $1 \leqslant k \leqslant n-1$.

We use shift lacunarity sequences to 'localize' the actions of Toeplitz operators $\tilde{T}_{a}$. Denote by $P_{i}$ the orthogonal projection onto $F_{i}$. Observe that

$$
\left.\begin{array}{rlrl}
S^{m p} S^{* m q} \xi_{i} & =S^{m(p-q)} \xi_{i} & & \text { if } p+q \leqslant 2\left(K_{i}+1\right), \\
\left(I-P_{i}\right) S^{m p} S^{* m q} \xi_{i} & =0 & & \text { if } p+q \leqslant K_{i}, \\
\left\langle S^{m p} S^{* m q} \xi_{i}, \xi_{k}\right\rangle & =0 & & \text { if } p+q \leqslant \max \left\{K_{i}, K_{k}\right\}
\end{array}\right\}
$$

(here $p$ and $q$ are non-negative integers).

We begin by showing that 'badly off-diagonal' parts of operators can be neglected.

Lemma 3.5. Suppose that $\left(\xi_{i}\right)$ is a shift lacunary normalized sequence in $X(C, m)=$ $X, Z=\operatorname{span}\left[\xi_{i} \mid i \in \mathbb{N}\right]$ and $T \in \mathrm{CB}(Z, X)$. Then, in the above notation, $W=T-$ $\left.\sum_{i=1}^{\infty} P_{i} T P_{i}\right|_{Z}$ is a Hilbert-Schmidt operator, satisfying $\|W\|_{2} \leqslant 16\|T\|_{\mathrm{cb}}$. 
Proof. For $n \in \mathbb{N}$ let $Q_{n}=\sum_{i=1}^{n} P_{i}$. By Lemma 2.1, it suffices to show that

$$
\left\|\left.Q_{n} W Q_{n}\right|_{Z}\right\|_{2} \leqslant 16\|T\|_{\mathrm{cb}} \text { for any } n \text {. }
$$

A simple argument shows that

$$
Q_{n} W Q_{n}=4 \cdot 2^{-n} \sum_{\mathcal{F} \subset\{1,2, \ldots, n\}}\left(Q_{n}-P_{\mathcal{F}}\right) W P_{\mathcal{F}}=4 \cdot 2^{-n} \sum_{\mathcal{F} \subset\{1,2, \ldots, n\}}\left(Q_{n}-P_{\mathcal{F}}\right) T P_{\mathcal{F}},
$$

where $P_{\mathcal{F}}=\sum_{i \in \mathcal{F}} P_{i}$. Thus, it remains to prove that, for any $\mathcal{F}, c \leqslant 4\|T\|_{\mathrm{cb}}$, where $c=\left\|\left.\left(Q_{n}-P_{\mathcal{F}}\right) T P_{\mathcal{F}}\right|_{Z}\right\|_{2}$. Define an isometry $v: \ell_{2}(\mathcal{F}) \rightarrow Z$ by setting $v e_{i}=\xi_{i}$ (the vectors $\left(e_{i}\right)_{i \in \mathcal{F}}$ form the canonical basis of $\left.\ell_{2}(\mathcal{F})\right)$. Find an operator $u: X \rightarrow \ell_{2}(\mathcal{F})$ such that $\|u\|_{2}=1, u\left(Q_{n}-P_{\mathcal{F}}\right)=u$ and $\left\|u\left(Q_{n}-P_{\mathcal{F}}\right) T P_{\mathcal{F}} v\right\|_{1}=c$.

To estimate $C^{-(p+q)}\left\|u S^{p m} S^{* q m} v\right\|_{1}$, observe that

$$
\left\|u S^{p m} S^{* q m} v\right\|_{1} \leqslant \sum_{i \in \mathcal{F}}\left\|u S^{p m} S^{* q m} v e_{i}\right\|=\sum_{i \in \mathcal{F}}\left\|u\left(Q_{n}-P_{\mathcal{F}}\right) S^{p m} S^{* q m} \xi_{i}\right\| .
$$

However, by (3.1), $\left(Q_{n}-P_{\mathcal{F}}\right) S^{m p} S^{* m q} \xi_{i}=0$ if $p+q \leqslant i / \alpha$. For other values of $j$, we use the trivial estimate $\left\|u\left(Q_{n}-P_{\mathcal{F}}\right) S^{m p} S^{* m q} \xi_{i}\right\| \leqslant 1$. Therefore,

$$
\sum_{i \in \mathcal{F}}\left\|u\left(Q_{n}-P_{\mathcal{F}}\right) S^{m p} S^{* m q} \xi_{i}\right\| \leqslant|\{i \in \mathcal{F} \mid i<\alpha(p+q)\}| \leqslant 1+\alpha(p+q) \leqslant \mathrm{e}^{\alpha(p+q)} .
$$

By our choice of $\alpha, C \leqslant \mathrm{e}^{2 \alpha}$; hence, $C^{-(p+q)}\left\|u S^{m p} S^{* m q} v\right\|_{1} \leqslant 1$ for all values of $p$ and $q$. By Lemma 2.2, $\|T\|_{\mathrm{cb}} \geqslant \frac{1}{4} c$.

Note that any c.b. map on the space $Z$ is a Hilbert-Schmidt perturbation of a scalar (see $[\mathbf{2 1}]$ ).

Corollary 3.6. Suppose that $Z$ is as in Lemma 3.5 and $T \in \mathrm{CB}(Z)$. There then exists a Hilbert-Schmidt operator $W \in B(Z)$, such that $\|W\|_{2} \leqslant 16\|T\|_{\mathrm{cb}}$, and $\Lambda=T-W$ is a diagonal operator (that is, $\Lambda \xi_{i}=\lambda_{i} \xi_{i}$ for any $i$, with $\lambda_{i} \in \mathbb{C}$ ).

Proof. Note that $T \xi_{j}=\sum_{i=1}^{\infty}\left\langle T \xi_{j}, \xi_{i}\right\rangle \xi_{i}$ for any $j$. By definition, $P_{i} \xi_{j}=\delta_{i j} \xi_{j}\left(\delta_{i j}\right.$ is Kronecker's delta). Therefore, $\sum_{i=1}^{\infty} P_{i} T P_{i} \xi_{j}=\lambda_{j} \xi_{j}$, where $\lambda_{j}=\left\langle T \xi_{j}, \xi_{j}\right\rangle$. Let $\Lambda$ be the diagonal operator on $Z$ corresponding to $\left(\lambda_{j}\right)_{j \in \mathbb{N}}$, and note that $\Lambda=\left.\sum_{i=1}^{\infty} P_{i} T P_{i}\right|_{Z}$. An application of Lemma 3.5 completes the proof.

Next we show that, for any $n \geqslant 0$, every $T \in \mathrm{CB}(Z, X)$ (with $Z$ as above) can be approximated 'reasonably well' by linear combinations of $S^{m j}$, with $|j| \leqslant n$.

Lemma 3.7. Suppose a normalized sequence $\left(\xi_{i}\right)_{i \in \mathbb{N}}$ is shift lacunary, and let $Z=$ $\operatorname{span}\left[\xi_{i} \mid i \in \mathbb{N}\right]$. For $N \in \mathbb{N}$ let $Z_{N}=\operatorname{span}\left[\xi_{i} \mid i>N\right]$. Then for every $T \in \operatorname{CB}(Z, X)$ and every $n \geqslant 0$ there exist complex numbers $\left(a_{j}\right)_{|j| \leqslant n}$, such that

$$
\sum_{j}\left|a_{j}\right| C^{|j|} \leqslant 4 \inf _{N}\left\|\left.T\right|_{Z_{N}}\right\|_{\mathrm{cb}}
$$


and

$$
\inf _{N}\left\|\left.\left(T-\sum_{j} a_{j} S^{m j}\right)\right|_{Z_{N}}\right\| \leqslant 4 C^{-(n+1)} \inf _{N}\left\|\left.T\right|_{Z_{N}}\right\|_{\mathrm{cb}} .
$$

Proof. By Lemma 3.5, $\left.\sum_{i} P_{i} T P_{i}\right|_{Z}$ is a c.b. map, and $T-\left.\sum_{i} P_{i} T P_{i}\right|_{Z}$ is HilbertSchmidt. By Lemma 2.1,

$$
\inf _{N}\left\|\left.\left(T-\sum_{i} P_{i} T P_{i}\right)\right|_{Z_{N}}\right\|_{\mathrm{cb}} \leqslant \inf _{N}\left\|\left.\left(T-\sum_{i} P_{i} T P_{i}\right)\right|_{Z_{N}}\right\|_{2}=0 .
$$

Therefore,

$$
\inf _{N}\left\|\left.T\right|_{Z_{N}}\right\|_{\mathrm{cb}}=\inf _{N}\left\|\left.\left(\sum_{i} P_{i} T P_{i}\right)\right|_{Z_{N}}\right\|_{\mathrm{cb}} .
$$

Thus, it suffices to show that, for any $T=\left.\sum_{i} P_{i} T P_{i}\right|_{Z} \in \mathrm{CB}(Z, X)$ such that $\left\|\left.T\right|_{Z_{K}}\right\|_{\mathrm{cb}}<$ $\frac{1}{4}$ for some $K$, we have $\inf _{N>n} c_{n, N} \leqslant C^{-(n+1)}$, where

$$
c_{n, N}=\inf \left\{\left\|\left.\left(\sum_{j=-n}^{n} a_{j} S^{m j}-T\right)\right|_{Z_{N}}\right\|\left|\sum_{j=-n}^{n}\right| a_{j} \mid C^{|j|} \leqslant 1\right\} .
$$

Denote by $\mathcal{P}_{N}$ the set of operators $W: Z_{N} \rightarrow X$ such that $W \xi_{i} \in F_{i}$ for any $i>N$ (we equip $\mathcal{P}_{N}$ with the norm inherited from $B\left(Z_{N}, X\right)$ ). By our assumption, $\left.T\right|_{Z_{N}} \in \mathcal{P}_{N}$ for any $N$. Moreover, the definition of shift lacunarity implies that $\left.S^{m j}\right|_{Z_{N}} \in \mathcal{P}_{N}$ if $|j| \leqslant N$. Therefore,

$$
\left.\left(\sum_{j=-n}^{n} a_{j} S^{m j}-T\right)\right|_{Z_{N}} \in \mathcal{P}_{N} \text { for } N \geqslant n
$$

It is easy to check that $\mathcal{P}_{N}$ is a Banach space, which is isometric to the $\ell_{\infty}$ direct sum $\left(\bigoplus_{i>N} F_{i}\right)_{\infty}$. Indeed, the ranges of the projections $P_{i}$ are mutually orthogonal; hence, the map Id : $\mathcal{P}_{N} \rightarrow\left(\bigoplus_{i} F_{i}\right)_{\infty}: W \mapsto\left(W \xi_{i}\right)_{i>N}$ is an isometry.

Suppose, for the sake of contradiction, that $\inf _{N} c_{N, n}>C^{-(n+1)}$. There then exists $c>C^{-(n+1)}$ such that $\left.T\right|_{Z_{N}} \notin Y_{n, N}$ for any $N>\max \{n, K\}$. Here, $B_{\mathcal{P}_{N}}$ is the closed unit ball of $\mathcal{P}_{N}$, 'conv' denotes the closed convex hull of a set, and

$$
Y_{n, N}=c C^{n+1} \operatorname{conv}\left(C^{-(n+1)} B_{\mathcal{P}_{N}} \cup\left\{\sum_{j=-n}^{n} a_{j} S^{m j}\left|Z_{N}\right| \sum_{j=-n}^{n}\left|a_{j}\right| C^{|j|} \leqslant 1\right\}\right) .
$$

By the Hahn-Banach theorem, there exists $f_{0} \in \mathcal{P}_{N}^{*}$ such that $\left|f_{0}\left(\left.T\right|_{Z_{N}}\right)\right|>c$, and $\left|f_{0}(W)\right|<c$ for any $W \in Y_{n, N}$. Consequently, $\left\|f_{0}\right\|<1$, and $\left|f_{0}\left(\left.S^{m j}\right|_{Z_{N}}\right)\right|<C^{|j|-n-1}$ if $|j| \leqslant n$. By Helly's theorem (or by the local reflexivity principle), there exists $f \in\left(\mathcal{P}_{N}\right)_{*}$ such that $\|f\|<1$, and

$$
f\left(\left.S^{m j}\right|_{Z_{N}}\right)=f_{0}\left(\left.S^{m j}\right|_{Z_{N}}\right) \quad \text { if }|j| \leqslant n
$$


(consequently, $\left|f\left(S^{m j} \mid Z_{N}\right)\right|<C^{|j|-n-1}$ for such $j$ ). Here, we identify $\left(\mathcal{P}_{N}\right)_{*}$ (a predual of $\left.\mathcal{P}_{N}\right)$ with $\left(\bigoplus_{i>N} F_{i}\right)_{1}$. Thus, $f=\left(\eta_{i}\right)_{i>N}$, with $\eta_{i} \in F_{i}$, and $\sum_{i>N}\left\|\eta_{i}\right\|=\|f\|<1$. The action of $f$ on $\mathcal{P}_{N}$ is defined by setting

$$
f(W)=\sum_{i>N}\left\langle W \xi_{i}, \eta_{i}\right\rangle \quad \text { for } W \in \mathcal{P}_{N} .
$$

Find a sequence $\max \{n, K\}<N_{0}<N_{1}<N_{2}<\cdots$ of positive integers and a sequence $\left(f_{k}\right)_{k=1}^{\infty}$ such that, for every $k$,

$$
\begin{gathered}
f_{k}=\left(b_{i}^{2} \eta_{i}\right)_{i=N_{k-1}}^{N_{k}-1}, \quad \text { where } \eta_{i} \in F_{i},\left\|\eta_{i}\right\|=1, b_{i} \geqslant 0, \sum_{i=N_{k-1}}^{N_{k}-1} b_{i}^{2}<1, \\
\left|\sum_{i=N_{k-1}}^{N_{k}-1} b_{i}^{2}\left\langle T \xi_{i}, \eta_{i}\right\rangle\right|>c \text { and }\left|\sum_{i=N_{k-1}}^{N_{k}-1} b_{i}^{2}\left\langle S^{m j} \xi_{i}, \eta_{i}\right\rangle\right|<C^{|j|-n-1} \text { when }|j| \leqslant n .
\end{gathered}
$$

Select $M>C^{2(n+1)}$, and define the operators $v: \ell_{2}^{M} \rightarrow Z_{N_{0}}$ and $u: X \rightarrow \ell_{2}^{M}$ by setting

$$
v e_{k}=\sum_{i=N_{k-1}}^{N_{k}-1} b_{i} \xi_{i} \text { and } u^{*} e_{k}=\frac{1}{\sqrt{M}} \sum_{i=N_{k-1}}^{N_{k}-1} b_{i} \eta_{i}
$$

(as usual, $\left(e_{i}\right)_{i=1}^{M}$ is the canonical basis from $\ell_{2}^{M}$ ). Then $\|u\|_{2} \leqslant 1$ and $\|v\| \leqslant 1$. Moreover,

$$
\left|\left\langle\left. u T\right|_{Z_{N_{0}}} v e_{k}, e_{k}\right\rangle\right|=\frac{1}{\sqrt{M}}\left|\sum_{i=N_{k-1}}^{N_{k}-1} b_{i}^{2}\left\langle T \xi_{i}, \eta_{i}\right\rangle\right|>\frac{c}{\sqrt{M}}
$$

for $1 \leqslant k \leqslant M$; hence, $\|u T v\|_{1} \geqslant c \sqrt{M}$. It remains to estimate $C^{-(p+q)}\left\|u S^{m p} S^{* m q} v\right\|_{1}$ $(p, q \geqslant 0)$ from above. For $p+q>n$, we have

$$
C^{-(p+q)}\left\|u S^{m p} S^{* m q} v\right\|_{1} \leqslant C^{-(p+q)} \sqrt{M} \leqslant C^{-(n+1)} \sqrt{M} .
$$

If $p+q \leqslant n$, observe that, by (3.1),

$$
\sqrt{M}\left|\left\langle u S^{m p} S^{* m q} v e_{k}, e_{k}\right\rangle\right|=\left|\sum_{i=N_{k-1}}^{N_{k}-1} b_{i}^{2}\left\langle S^{m(p-q)} \xi_{i}, \eta_{i}\right\rangle\right|<C^{|p-q|-n-1} \leqslant C^{(p+q)-n-1}
$$

for $1 \leqslant k \leqslant M$. Also by (3.1), $\left\langle u S^{m p} S^{* m q} v e_{k}, e_{\ell}\right\rangle=0$ if $k \neq \ell$. Therefore,

$$
\left\|u S^{m j} v\right\|_{1} \leqslant \sum_{k, \ell=1}^{M}\left|\left\langle u S^{m j} v e_{k}, e_{\ell}\right\rangle\right| \leqslant C^{-(n+1)} \sqrt{M} .
$$

By Lemma 2.2,

$$
\left\|\left.T\right|_{Z_{N_{0}}}\right\|_{\mathrm{cb}}>\frac{c \sqrt{M}}{4 \max \left\{C^{-(n+1)} \sqrt{M}, 1\right\}}>\frac{1}{4},
$$

which contradicts the assumption that $\left\|\left.T\right|_{Z_{N_{0}}}\right\|_{\mathrm{cb}}<\frac{1}{4}$. 
Lemma 3.8. Suppose that $Z$ and $T$ are as in Lemma 3.7. There then exists a sequence $a \in \ell_{1}\left(C^{|j|}, \mathbb{Z}\right)$ for which $\|a\|_{w} \leqslant 4\|T\|_{\mathrm{cb}}$, and $T-\left.\tilde{T}_{a}\right|_{Z}$ is compact.

Proof. Assume that $\|T\|_{\mathrm{cb}}<\frac{1}{4}$. By Lemma 3.7, for every $n \in \mathbb{N}$ there exist complex numbers $\left(a_{j}^{(n)}\right)_{j=-n}^{n}$ and $N_{n} \in \mathbb{N}$ such that

$$
\sum_{j=-n}^{n}\left|a_{j}^{(n)}\right| C^{|j|}<1 \text { and }\left\|\left.\left(\sum_{j=-n}^{n} a_{j}^{(n)} S^{m j}-T\right)\right|_{Z_{N_{n}}}\right\|<C^{-(n+1)}
$$

(we keep the notation of Lemma 3.7). Without loss of generality, we can assume that $N_{1}<N_{2}<\cdots$.

For $|j|>n$, let $a_{j}^{(n)}=0$, and regard $a^{(n)}=\left(a_{j}^{(n)}\right)_{j \in \mathbb{Z}}$ as an element of $\ell_{1}\left(C^{|j|}, \mathbb{Z}\right)$, with $\left\|a^{(n)}\right\|_{w}<1$. By passing to a subsequence if necessary, we can assume that the sequence $\left(a^{(n)}\right)$ converges weak ${ }^{*}$ to $a=\left(a_{j}\right)_{j \in \mathbb{Z}} \in \ell_{1}\left(C^{|j|}, \mathbb{Z}\right)$ (in other words, $\lim _{n} a_{j}^{(n)}=a_{j}$ for any $j \in \mathbb{Z}$ ). Clearly, $\|a\|_{w} \leqslant 1$. Moreover, $\lim _{n}\left\|\tilde{T}_{a}-\tilde{T}_{a^{(n)}}\right\|=0$. Indeed, fix $\varepsilon>0$, and find $K \in \mathbb{N}$ such that $C^{-(K+1)}<\frac{1}{3} \varepsilon$. Find $M \in \mathbb{N}$ such that $\left|a_{j}^{(n)}-a_{j}\right|<\varepsilon / 9 K$ for $|j| \leqslant K$ and $n \geqslant M$. For such $n$,

$$
\begin{aligned}
\left\|\tilde{T}_{a}-\tilde{T}_{a^{(n)}}\right\| & =\left\|\sum_{j \in \mathbb{Z}}\left(a_{j}-a_{j}^{(n)}\right) S^{m j}\right\| \\
& \leqslant \sum_{|j| \leqslant K}\left|a_{j}-a_{j}^{(n)}\right|+\sum_{|j|>K}\left(\left|a_{j}\right|+\left|a_{j}^{(n)}\right|\right) \\
& <(2 K+1) \frac{\varepsilon}{9 K}+\frac{2 \varepsilon}{3} \\
& <\varepsilon .
\end{aligned}
$$

Therefore,

$$
\left\|\left.\left(\tilde{T}_{a}-T\right)\right|_{Z_{N_{\ell}}}\right\| \leqslant\left\|\tilde{T}_{a}-\tilde{T}_{a^{(\ell)}}\right\|+\left\|\left.\left(\tilde{T}_{a^{(\ell)}}-T\right)\right|_{Z_{N_{\ell}}}\right\|<\varepsilon+C^{-\ell}
$$

for $\ell>\max \{K, M\}$. Thus, $\lim _{\ell}\left\|\left.\left(\tilde{T}_{a}-T\right)\right|_{Z_{N_{\ell}}}\right\|=0$, and hence $T-\left.\tilde{T}_{a}\right|_{Z}$ is compact.

Proof of Theorem 1.1 (ii). Suppose that $Y$ is an infinite-dimensional subspace of $X$ and that $T \in \mathrm{CB}(Y, X)$ satisfies $\|T\|_{\mathrm{cb}}<\frac{1}{4}$. First show that for every $c>0$ there exists $a \in \ell_{1}\left(C^{|j|}, \mathbb{Z}\right)$ such that $\|a\|_{w} \leqslant 1$, and $\lim _{N}\left\|\left.\left(\tilde{T}_{a}-T\right)\right|_{Y_{N}}\right\| \leqslant c$ (here $Y_{N}=Y \cap \operatorname{span}\left[\delta_{i} \mid\right.$ $i \geqslant N]$ ). Indeed, suppose this is false for some $c \in(0,1)$. Find a sequence $\left(a^{(n)}\right)$ which is dense in the unit ball of $\ell_{1}\left(C^{|j|}, \mathbb{Z}\right)$ and let $\tilde{T}^{(n)}=\tilde{T}_{a^{(n)}}$. For each $n, Y$ contains an infinite-dimensional subspace $Y_{n}$ such that $\left\|\left(\tilde{T}^{(n)}-T\right) \xi\right\|>\frac{1}{2} c$ for any $\xi \in Y_{n}$. By Proposition 3.4, there exists a shift lacunary sequence $\left(\xi_{i}\right)_{i \in \mathbb{N}}$ such that $\xi_{i} \in Y_{n}$ whenever $i \in\left\{2^{n-1}, 3 \cdot 2^{n-1}, 5 \cdot 2^{n-1}, \ldots\right\}$. Therefore, $\lim \sup _{i}\left\|\left(\tilde{T}_{a}-T\right) \xi_{i}\right\| \geqslant \frac{1}{2} c$ whenever $a$ belongs to the unit ball of $\ell_{1}\left(C^{|j|}, \mathbb{Z}\right)$, which contradicts Lemma 3.8 .

Thus, there exist a sequence of positive integers $N_{1}<N_{2}<\cdots$ and a sequence $\left(a^{(n)}\right)$ of elements of the unit ball of $\ell_{1}\left(C^{|j|}, \mathbb{Z}\right)$, such that $\left\|\left.\left(\tilde{T}^{(n)}-T\right)\right|_{Y_{N_{n}}}\right\|<2^{-n}$ for any $n \in \mathbb{N}$ (here, as before, $\tilde{T}^{(n)}=\tilde{T}_{a^{(n)}}$ ). Passing to a subsequence if necessary (see the proof of 
Lemma 3.8), we can assume the existence of $a \in \ell_{1}\left(C^{|j|}, \mathbb{Z}\right)$, such that $\|a\|_{w} \leqslant 1$, and $\lim _{n}\left\|\tilde{T}_{a}-\tilde{T}^{(n)}\right\|<2^{-n}$. Therefore,

$$
\left\|\left.\left(\tilde{T}_{a}-T\right)\right|_{Y_{N_{n}}}\right\| \leqslant\left\|\left.\left(\tilde{T}^{(n)}-T\right)\right|_{Y_{N_{n}}}\right\|+\left\|\tilde{T}_{a}-\tilde{T}^{(n)}\right\|<2^{1-n}
$$

for any $n \in \mathbb{N}$. Thus, $\left.\tilde{T}_{a}\right|_{Y}-T$ is compact.

Remark 3.9. If $Y$ is a coordinate subspace of $X$, then $a$ as above is unique. Indeed, if $Y=\operatorname{span}\left[\delta_{i_{k}} \mid k \in \mathbb{N}\right]$, then $a_{j}=\lim _{k \rightarrow \infty}\left\langle T \delta_{i_{k}}, \delta_{i_{k}+m j}\right\rangle$ for any $j \in \mathbb{Z}$. For a generic $Y \hookrightarrow X$ and $T \in \mathrm{CB}(Y, X)$, there may be more than one $a \in \ell_{1}\left(C^{|j|}, \mathbb{Z}\right)$ for which $T-\left.\tilde{T}_{a}\right|_{Y}$ is compact (see $\S 7.2$ for more details).

To prove Theorems 3.1 and 3.2 , we follow in the footsteps of [8], and associate complexvalued functions with members of $\operatorname{CB}(Y, X)$. To this end, identify $\ell_{1}\left(C^{|j|}, \mathbb{Z}\right)$ with the set $\mathcal{Y}$ of those analytic functions $f$ on the annulus $\mathbb{A}=\left\{z \in \mathbb{C}\left|C^{-1} \leqslant\right| z \mid \leqslant C\right\}$, whose Laurent coefficients belong to $\ell_{1}\left(C^{|j|}, \mathbb{Z}\right)$. More precisely, for $f \in \mathcal{Y}$ we have $f(z)=\sum_{j} a_{j} z^{j}$, with $\left\|\left(a_{j}\right)\right\|_{w}<\infty$. Let $\|f\|_{\mathcal{Y}}=\left\|\left(a_{j}\right)\right\|_{w}$. Clearly, $\mathcal{Y}$ (equipped with pointwise multiplication) is isometric, and algebraically isomorphic, to $\ell_{1}\left(C^{|j|}, \mathbb{Z}\right)$ with its convolution.

Suppose that $Y$ is an infinite-dimensional coordinate subspace of $X$ and that $T \in$ $\mathrm{CB}(Y, X)$. By Theorem 1.1 (ii) and Remark 3.9, there exists a unique $a=\left(a_{j}\right)_{j \in \mathbb{Z}} \in$ $\ell_{1}\left(C^{|j|}, \mathbb{Z}\right)$ for which $T-\tilde{T}_{a}$ is compact. We construct a function $\phi(T) \in \mathcal{Y}$ by setting

$$
\phi(T)(z)=\sum_{j} a_{j} z^{j} \quad \text { for } z \in \mathbb{A} .
$$

Note that, if $Y_{1}$ and $Y_{2}$ are infinite-dimensional coordinate subspaces of $X, T_{1} \in$ $\mathrm{CB}\left(Y_{1}, Y_{2}\right)$ and $T_{2} \in \mathrm{CB}\left(Y_{2}, X\right)$, then $\phi\left(T_{2} T_{1}\right)=\phi\left(T_{1}\right) \phi\left(T_{2}\right)$.

Thus, we can pass from $\mathrm{CB}(X)$ to the well-understood, commutative Banach algebra $\mathcal{Y}$ (see $[\mathbf{4}$, Chapter 4.6] for its properties). This allows us to investigate c.b. projections on, and complete isomorphisms of, the space $X$.

Proof of Theorem 3.1. Suppose that $Y$ is an infinite-dimensional coordinate subspace of $X, T \in \mathrm{CB}(Y)$ and $T^{2}=T$. Let $f=\phi(T)$. By the reasoning above, $f^{2}=f$. That is, $f(z)=0$ or $f(z)=1$ for any $z \in \mathbb{A}$. However, $f$ must be continuous, and hence either $f \equiv 0$, or $f \equiv 1$. In the former case, $T$ is compact, and hence $\operatorname{ran} T$ is finite dimensional. In the second case, $I-T$ is compact, and hence $\operatorname{ran}(I-T)$ is finite dimensional. Therefore, $\operatorname{ker} T$ is finite dimensional.

Remark 3.10. As we show in $\S 7.1$, the statement of Theorem 3.1 fails for non-coordinate subspaces of $X$.

The next two lemmas allow us to prove a statement more general than Theorem 3.2. These results will be used again to compute the $K$-groups of $\mathrm{CB}(X)$ in $\S 6$.

Below, we define the direct sum $Y \oplus Z$ ( $Y$ and $Z$ are operator spaces), for $y \in M_{n}(Y)$ and $z \in M_{n}(Z)$, by setting

$$
\|y \oplus z\|_{M_{n}(Y \oplus Z)}=\max \left\{\|y\|_{M_{n}(Y)},\|z\|_{M_{n}(Z)},\|y \oplus z\|_{M_{n}\left(\operatorname{MIN}\left(Y \oplus_{2} Z\right)\right)}\right\},
$$


where $\operatorname{MIN}(E)$ denotes the Banach space $E$, equipped with its minimal operator space structure. In the following, $Y^{n}$ stands for $Y \oplus Y \oplus \cdots \oplus Y$ ( $n$ times). If $n=0$, we identify $Y^{n}$ with $\{0\} . B\left(Y^{n}\right)$ is henceforth identified with $M_{n}(B(Y))$ (the space of $n \times n$ matrices with entries in $B(Y))$ as a vector space. Similarly, we identify $\mathrm{CB}\left(Y^{n}\right)$ with $M_{n}(\mathrm{CB}(Y))$.

Lemma 3.11. Suppose that $Y$ is an infinite codimensional subspace of $X^{n}, n \geqslant 1$. Then no completely bounded map from $X^{n}$ into $Y$ has a bounded inverse.

Proof. An operator $T \in \mathrm{CB}\left(X^{n}\right)$ can be represented as an $n \times n$ matrix $\left(T^{(p q)}\right)_{p, q=1}^{n}$, whose entries are c.b. maps on $X$. By Theorem 1.1 (ii),

$$
T^{(p q)}=\tilde{T}_{a(p q)}+W^{(p q)}
$$

with $W^{(p q)}$ compact, and

$$
a^{(p q)}=\left(a_{j}^{(p q)}\right)_{j \in \mathbb{Z}} \in \ell_{1}\left(C^{|j|}, \mathbb{Z}\right) .
$$

Denote the operator $\left(\tilde{T}_{a^{(p q)}}\right)_{p, q=1}^{n}$ by $\tilde{T}_{a}$, where $a=\left(a_{j}\right)_{j \in \mathbb{Z}}$, and $a_{j}=\left(a_{j}^{(p q)}\right)_{p, q=1}^{n}$ is an $n \times n$ matrix for any $j$; clearly,

$$
\sum_{j \in \mathbb{Z}} C^{|j|}\left\|a_{j}\right\|<\infty
$$

Similarly, denote the operator $\left(W^{(p q)}\right)_{p, q=1}^{n}$ by $W$.

Consider a function $f=\left(I_{M_{n}} \otimes \phi\right)(T): \mathbb{A} \mapsto M_{n}$. That is, $f=\left(f^{(p q)}\right)_{p, q=1}^{n}$, with $f^{(p q)}=\phi\left(T^{(p q)}\right)$ or, in other words,

$$
f(z)=\sum_{j} a_{j} z^{m j}
$$

where $a_{j}=\left(a_{j}^{(p q)}\right)_{p, q=1}^{n}$ is an $n \times n$ matrix. Suppose first that det $f(z)=0$ for some $z \in \mathbb{T}=\{\omega \in \mathbb{C}|| \omega \mid=1\}$. Fix $\varepsilon>0$, and show that there exists a unit vector $\xi \in X^{n}$ with finite support, such that $\left\|T\left(I_{\ell_{2}^{n}} \otimes S\right)^{\ell} \xi\right\|<\varepsilon$ for any $\ell \geqslant 0$ (here $I_{\ell_{2}^{n}} \otimes S \in B\left(X^{n}\right)$ is defined via $I_{\ell_{2}^{n}} \otimes S\left(\xi_{1}, \ldots, \xi_{n}\right)=\left(S \xi_{1}, \ldots, S \xi_{n}\right)$ for $\left.\xi_{1}, \ldots, \xi_{n} \in X\right)$. This would imply that $T$ does not have a bounded inverse.

Denote by $\boldsymbol{M}_{f}$ the operator of multiplication by $f$, acting on $\ell_{2}^{n}\left(L_{2}(\mathbb{T})\right)$. By the density of trigonometric polynomials, there exists

$$
p(z)=\sum_{|k| \leqslant N} b_{k} z^{k}, \quad b_{k} \in \ell_{2}^{n},
$$

such that $\|p\|_{\ell_{2}^{n}\left(L_{2}(\mathbb{T})\right)}=1$, and $\left\|\boldsymbol{M}_{f} p\right\|_{\ell_{2}^{n}\left(L_{2}(\mathbb{T})\right)}<\frac{1}{2} \varepsilon$. Let $\eta=\sum_{|k| \leqslant N} b_{k} \otimes \delta_{k+N}$, and note that $\left\|\tilde{T}_{a}\left(I_{\ell_{2}^{n}} \otimes S\right)^{\ell} \eta\right\|<\frac{1}{2} \varepsilon$ for any $\ell \geqslant 0$. By the compactness of $W$, there exists $K \geqslant 0$ such that $\left\|W\left(I_{\ell_{2}^{n}} \otimes S\right)^{\ell} \eta\right\|<\frac{1}{2} \varepsilon$ for any $\ell \geqslant K$. Let $\xi=\left(I_{\ell_{2}^{n}} \otimes S\right)^{K} \eta$. Then $\left\|T\left(I_{\ell_{2}^{n}} \otimes S\right)^{\ell} \xi\right\|<\varepsilon$ for any $\ell \geqslant 0$.

If $\operatorname{det} f$ does not vanish on $\mathbb{T}$, then the operator $\tilde{T}_{a}$ is Fredholm (see [5, Chapter 1$]$ or [15]); hence, so is $T=\tilde{T}_{a}+W$. In particular, $\operatorname{dim}\left(X^{n} / T\left(X^{n}\right)\right)<\infty$. 
Corollary 3.12. Suppose that $X_{1}$ is a finite codimensional subspace of $X^{n}, n \geqslant 1$, and $Y$ is an infinite codimensional subspace of $X^{n}$. Then no completely bounded map from $X_{1}$ into $Y$ has a bounded inverse.

Proof. Consider $T \in \mathrm{CB}\left(X_{1}, Y\right)$. Find a finite-dimensional $E \hookrightarrow X^{n}$ such that $X^{n}=$ $\operatorname{span}\left[E, X_{1}\right]$ and $E \cap X_{1}=\{0\}$. Find $F \hookrightarrow X^{n}$ such that $\operatorname{dim} E=\operatorname{dim} F$, and $F \cap Y=\{0\}$. There then exists an invertible operator $U: E \rightarrow F$. Let $T^{\prime}=T P+U(I-P)$, where $P$ is a projection for which $\operatorname{ker} P=E, \operatorname{ran} P=X_{1}$. Clearly, $T^{\prime} \in \mathrm{CB}\left(X^{n}, \operatorname{span}[Y, F]\right)$. If $T$ has a bounded inverse, then so has $T^{\prime}$, which contradicts Lemma 3.11.

Lemma 3.13. Suppose that $n \geqslant 1, X_{1}$ and $Y$ are subspaces of $X^{n}$ and that $\operatorname{dim} X^{n} / X_{1}<\infty$. Then $X_{1}$ is completely isomorphic to $Y$ if and only if $\operatorname{dim} X^{n} / Y$ is finite, and $m$ divides $\operatorname{dim} X^{n} / X_{1}-\operatorname{dim} X^{n} / Y$.

Proof. By the construction of $X(2.1)$, the operators $S^{m}$ and $S^{* m}$ are completely bounded. Hence, any coordinate subspace of $X$ is completely isomorphic to its subspaces of codimension $m, 2 m, 3 m, \ldots$. Since all subspaces of $X^{n}$ of given (finite) codimension are completely isomorphic, we have shown that $X_{1}$ and $Y$ are completely isomorphic if $\operatorname{dim} X^{n} / X_{1}-\operatorname{dim} X^{n} / Y$ is divisible by $m$.

Suppose, on the other hand, that $X_{1}$ and $Y$ are subspaces of $X^{n}$, that $X_{1}$ is completely isomorphic to $Y$ and that $\operatorname{dim} X / X_{1}<\infty$. By Corollary 3.12, $\operatorname{dim} X^{n} / Y<\infty$. Suppose, without loss of generality, that $\operatorname{dim} X^{n} / X_{1} \leqslant \operatorname{dim} X^{n} / Y$, and $T: X_{1} \rightarrow Y$ is a complete isomorphism. By adding a finite-rank operator if necessary (as in the proof of Corollary 3.12), we can view $T$ as a complete isomorphism from $X^{n}$ onto its subspace of finite codimension.

As in the proof of Lemma 3.11, consider a function $f=\left(I_{M_{n}} \otimes \phi\right)(T): \mathbb{A} \mapsto M_{n}$, corresponding to $a \in M_{n} \otimes \ell_{1}\left(C^{|j|}, \mathbb{Z}\right)$. Then $W=T-\tilde{T}_{a}$ is compact. $T$ is a Fredholm operator of index $K=\operatorname{dim} X / X_{1}-\operatorname{dim} X / Y$, and hence so is $\tilde{T}_{a}$. By [5] (or [15]), $-K$ is equal to the winding number of the function $z \mapsto \operatorname{det} f\left(z^{m}\right)$ around 0 , which must be divisible by $m$.

Proof of Theorem 3.2. Apply the previous lemma with $n=1$.

\section{When is $T-\tilde{T}_{a}$ Hilbert-Schmidt?}

In the previous section, we showed that every c.b. map from $Y$ to $X(Y \hookrightarrow X)$ is a compact perturbation of a Toeplitz operator $\tilde{T}_{a}$, with $a \in \ell_{1}\left(C^{|j|}, \mathbb{Z}\right)$. Below we show that, for certain subspaces, this perturbation is actually Hilbert-Schmidt. In particular, we establish this for $Y=X$ (Theorem 1.1(i) (b)). In addition, we prove the following theorem.

Theorem 4.1. Any infinite-dimensional subspace $Y$ of $X$ has an infinite-dimensional subspace $Z$ such that any $T \in \mathrm{CB}(Z, X)$ can be written as $T=\left.\tilde{T}_{a}\right|_{Z}+W$, with $a \in$ $\ell_{1}\left(C^{|j|}, \mathbb{Z}\right), W \in \mathcal{S}_{2},\|W\|_{2} \leqslant 20 \sqrt{2}\|T\|_{\mathrm{cb}}$, and $\|a\|_{w} \leqslant 4\|T\|_{\mathrm{cb}}$. Moreover, such a $Z$ can be selected so that any $T \in \mathrm{CB}(Z)$ can be written as $T=\lambda I_{Z}+W$, with $\lambda \in \mathbb{C}$, $W \in \mathcal{S}_{2},\|W\|_{2} \leqslant 700\|T\|_{\mathrm{cb}}$ and $|\lambda| \leqslant\|T\|$. In fact, any infinite-dimensional $Y$ contains 
an uncountable family of subspaces $Z$, which have the property described above, and are not completely isomorphic to each other.

To do this, we need a definition.

Definition 4.2. A sequence $\left(\xi_{i}\right)_{i \in \mathbb{N}} \subset X$ is called $c$-nice (here $c \geqslant 1$ ) if it is orthonormal and, for any $M \in \mathbb{N}$, any $\varepsilon>0$ and any finite set $\left(\eta_{k}\right)_{k=1}^{L} \in X$, there exists $N_{0}\left(\left(\eta_{k}\right)_{k=1}^{L}, \varepsilon, M\right) \in \mathbb{N}$ with the property that, for every $N \geqslant N_{0}\left(\left(\eta_{k}\right)_{k=1}^{L}, \varepsilon, M\right)$, there exists an orthogonal projection $Q$ for which the following conditions apply.

(i) $\left\|Q S^{m p} S^{* m q} v\right\|_{2} \leqslant c C^{p+q}$ for any $p, q \geqslant 0$. Here, $v: \ell_{2}^{M} \rightarrow \operatorname{span}\left[\xi_{i} \mid i \in \mathbb{N}\right]$ is defined by setting $v e_{i}=\left(\xi_{i}+\xi_{i+N}\right) / \sqrt{2}$ (as usual, $\left(e_{i}\right)_{i=1}^{M}$ is the canonical basis for $\ell_{2}^{M}$ ).

(ii) $\left\|Q \eta_{k}\right\| \geqslant\left(\left\|\eta_{k}\right\|-\varepsilon\right) / \sqrt{2}$ for $1 \leqslant k \leqslant L$.

A subspace $Y$ of $X$ is called $c$-nice if it has a $c$-nice orthonormal basis.

Ubiquity of $c$-nice sequences will be proved below (Lemmas 4.4 and 4.8). First we present a result underscoring their importance for this section.

Lemma 4.3. Suppose that $Y$ is a c-nice subspace of $X$ and that $T \in \operatorname{CB}(Y, X)$ is compact. Then $T$ is Hilbert-Schmidt, with $\|T\|_{2} \leqslant 4 \sqrt{2} c\|T\|_{\mathrm{cb}}$.

Proof. It suffices to show that, for every $\varepsilon>0$ and $M \in \mathbb{N}$,

$$
\|T\|_{\mathrm{cb}} \geqslant \frac{1}{4 c}\left(\sum_{i=1}^{M} \frac{\left(\left\|T \xi_{i}\right\|-\varepsilon\right)^{2}}{2}\right)^{1 / 2}
$$

where $\left(\xi_{i}\right)$ is a $c$-nice orthonormal basis in $Y$.

Taking $N>N_{0}\left(\left(T \xi_{i}\right)_{i=1}^{M}, \varepsilon, M\right)$ to be sufficiently large, and perturbing $T$ slightly, we can assume that $T \xi_{i+N}=0$ for $1 \leqslant i \leqslant M$. Let $v$ be as in the definition of niceness, and find $u: X \rightarrow \ell_{2}^{M}$ such that $u=u Q,\|u\|_{2}=1$ and

$$
\|u Q T v\|_{1}=\|Q T v\|_{2}=\left(\sum_{i=1}^{M}\left\|Q T v e_{i}\right\|^{2}\right)^{1 / 2} \geqslant\left(\sum_{i=1}^{M} \frac{\left(\left\|T \xi_{i}\right\|-\varepsilon\right)^{2}}{2}\right)^{1 / 2} .
$$

By the definition of niceness again,

$$
\left\|u S^{m p} S^{* m q} v\right\|_{1}=\left\|u Q S^{m p} S^{* m q} v\right\|_{1} \leqslant\left\|Q S^{m p} S^{* m q} v\right\|_{2} \leqslant c C^{p+q} .
$$

An application of Lemma 2.2 establishes (4.1).

Next we give examples of $c$-nice sequences. We say that a sequence $\left(\xi_{i}\right)_{i \in \mathbb{N}}$ is regular if it is normalized, shift lacunary, and there exists a sequence $\left(\alpha_{j}\right)_{j \geqslant 0}$ of complex numbers, such that

$$
\left|\left\langle S^{m j} \xi_{i}, \xi_{i}\right\rangle-\alpha_{j}\right|<\frac{\alpha^{2 i}}{2^{2 i+4}(1+i / \alpha)^{4}}
$$


if $1 \leqslant j \leqslant 2(1+i / \alpha)$ (here, as before, $\alpha=\min \{1, \ln C\} / 2)$. Note that, for $-1 \geqslant j \geqslant$ $-2(1+i / \alpha)$

$$
\left\langle S^{m j} \xi_{i}, \xi_{i}\right\rangle=\overline{\left\langle\xi_{i}, S^{m j} \xi_{i}\right\rangle}=\overline{\left\langle S^{-m j} \xi_{i}, \xi_{i}\right\rangle} .
$$

Therefore, defining $\alpha_{0}=1$ and $\alpha_{j}=\bar{\alpha}_{-j}$ for $j<0$, we conclude that, for a regular sequence, we have

$$
\left|\left\langle S^{m j} \xi_{i}, \xi_{i}\right\rangle-\alpha_{j}\right|<\frac{\alpha^{2 i}}{2^{2 i+4}(1+i / \alpha)^{4}} \quad \text { whenever }|j| \leqslant 2(1+i / \alpha) .
$$

Lemma 4.4. Every infinite-dimensional subspace of $\ell_{2}$ contains a regular sequence.

Proof. Suppose that $Y$ is an infinite-dimensional subspace of $X$. By Proposition 3.4, it contains a shift lacunary sequence $\left(\xi_{i}\right)_{i \in \mathbb{N}}$. By a diagonalization argument (and passing to a subsequence if necessary), we can assume that $\alpha_{j}=\lim _{k}\left\langle S^{m j} \xi_{i}, \xi_{i}\right\rangle$ exists for each $j>0$. Passing to a further subsequence, we can assure ourselves that the appropriate bound for $\left|\left\langle S^{m j} \xi_{i}, \xi_{i}\right\rangle-\alpha_{j}\right|$ holds if $1 \leqslant j \leqslant 2(1+i / \alpha)$.

Lemma 4.5. Any regular sequence is 1-nice.

Proof. Suppose, for $1 \leqslant k \leqslant L$, that $\eta_{k} \in X,\left\|\eta_{k}\right\|=1$ and $\operatorname{supp} \eta_{k} \subset\left[1, N_{0}\right]$. We shall show that, for any $N>\max \left\{N_{0}, M\right\}$, there exists an orthogonal projection $Q$ such that $\left\|Q \eta_{k}\right\| \geqslant\left\|\eta_{k}\right\| / \sqrt{2}$, and $\left\|Q S^{m p} S^{* m q} v\right\|_{2} \leqslant C^{p+q}$ for any $p, q \geqslant 0$ (as before, $\left(e_{i}\right)_{i=1}^{M}$ is the canonical basis for $\ell_{2}^{M}$, and $\left.v e_{i}=\left(\xi_{i}+\xi_{i+N}\right) / \sqrt{2}\right)$.

For $1 \leqslant i \leqslant M$ define the operators $w_{i}: \ell_{2}^{2 K_{i}+1} \rightarrow F_{i}$ and $w_{i}^{\prime}: \ell_{2}^{2 K_{i}+1} \rightarrow F_{i+N}$ by setting $w_{i} f_{j}=S^{m j} \xi_{i}$ and $w_{i}^{\prime} f_{j}=S^{m j} \xi_{i+N}$ (here $K_{i}=\lfloor i / \alpha\rfloor,\left(f_{j}\right)_{j=-K_{i}}^{K_{i}}$ is an orthonormal basis in $\ell_{2}^{2 K_{i}+1}$, and $F_{1}, F_{2}, \ldots$ are as in $\S 3$ ). Consider the polar decompositions $w_{i}=U_{i} A_{i}$ and $w_{i}^{\prime}=U_{i}^{\prime} A_{i}^{\prime}$, where $U_{i}$ and $U_{i}^{\prime}$ are isometries from $\ell_{2}^{2 K_{i}+1}$ into $F_{i}$ and $F_{i+N}$, respectively, and $A_{i}=\left(w_{i}^{*} w_{i}\right)^{1 / 2}, A_{i}^{\prime}=\left(w_{i}^{\prime *} w_{i}^{\prime}\right)^{1 / 2}$ act on $\ell_{2}^{2 K_{i}+1}$.

Now observe that, for $-K_{i} \leqslant j, k \leqslant K_{i}$,

$$
\left\langle w_{i}^{*} w_{i} f_{k}, f_{j}\right\rangle=\left\langle w_{i} f_{k}, w_{i} f_{j}\right\rangle=\left\langle S^{m k} \xi_{i}, S^{m j} \xi_{i}\right\rangle
$$

and therefore, $w_{i}^{*} w_{i}=\sum_{j, k=1}^{n}\left\langle S^{m(k-j)} \xi_{i}, \xi_{i}\right\rangle E_{j k}$ (here $E_{j k}$ is the matrix with 1 on the intersection of the $j$ th row and $k$ th column, and zeros elsewhere). A similar expansion can be written for $w_{i}^{\prime *} w_{i}^{\prime}$. By (4.2),

$$
\begin{aligned}
\mid\left\langle S^{m(k-j)}\right. & \left.\xi_{i}, \xi_{i}\right\rangle-\left\langle S^{m(k-j)} \xi_{i+N}, \xi_{i+N}\right\rangle \mid \\
& \leqslant\left|\left\langle S^{m(k-j)} \xi_{i}, \xi_{i}\right\rangle-\alpha_{k-j}\right|+\left|\left\langle S^{m(k-j)} \xi_{i+N}, \xi_{i+N}\right\rangle-\alpha_{k-j}\right| \\
& <\frac{\alpha^{2 i}}{2^{2 i+3}(1+i / \alpha)^{4}}
\end{aligned}
$$

for such $k$ and $j$, and hence

$$
\left\|w_{i}^{*} w_{i}-w_{i}^{\prime *} w_{i}^{\prime}\right\|_{2}^{2} \leqslant \sum_{j, k=0}^{K_{i}}\left|\left\langle S^{m(k-j)} \xi_{i}, \xi_{i}\right\rangle-\left\langle S^{m(k-j)} \xi_{i+N}, \xi_{i+N}\right\rangle\right|^{2} \leqslant \frac{\alpha^{4 i}}{2^{4 i+6} K_{i}^{2}} .
$$


Moreover, $\left\|w_{i}^{*} w_{i}+w_{i}^{\prime *} w_{i}^{\prime}\right\| \leqslant 2\left(2 K_{i}+1\right)$, and therefore, by [2, Theorem X.2.3],

$$
\left\|A_{i}-A_{i}^{\prime}\right\|^{2} \leqslant\left\|w_{i}^{*} w_{i}-w_{i}^{\prime *} w_{i}^{\prime}\right\|\left\|w_{i}^{*} w_{i}+w_{i}^{\prime *} w_{i}^{\prime}\right\| \leqslant\left(\frac{\alpha}{2}\right)^{2 i} .
$$

Now let $G_{i}=\operatorname{span}\left[\left(U_{i}+U_{i}^{\prime}\right) A_{i} f_{j} \mid-K_{i} \leqslant j \leqslant K_{i}\right]$ and $G=\operatorname{span}\left[G_{1}, \ldots, G_{M}\right]$. Note that $G_{i} \hookrightarrow F_{i}+F_{i+N}$, and hence the spaces $G_{i}$ are mutually orthogonal. We claim that the orthogonal projection $Q$, whose kernel is precisely $G$, satisfies the definition of 1-niceness. First note that any $\xi \in G$ can be written as $\xi=\xi_{1}+\xi_{2}$, with $\xi_{1} \in F_{1}+\cdots+F_{M}$, $\xi_{2} \in F_{N+1}+\cdots+F_{N+M}$, and $\left\|\xi_{1}\right\|=\left\|\xi_{2}\right\|=\|\xi\| / \sqrt{2}$. By the choice of $N,\left\langle\eta_{k}, \xi_{2}\right\rangle=0$ for $1 \leqslant k \leqslant L$, and hence $\left\|\eta_{k}-\xi\right\| \geqslant\left\|\eta_{k}\right\| / \sqrt{2}$ for any $\xi \in G$. Thus, $\left\|Q \eta_{k}\right\| \geqslant\left\|\eta_{k}\right\| / \sqrt{2}$.

Next we estimate

$$
\left\|Q S^{m p} S^{* m q} v\right\|_{2}^{2}=\sum_{i=1}^{M}\left\|Q S^{m p} S^{* m q} v e_{i}\right\|^{2}=\frac{1}{2} \sum_{k=1}^{M}\left\|Q S^{m p} S^{* m q}\left(\xi_{i}+\xi_{i+N}\right)\right\|^{2} .
$$

For $i<\alpha(p+q)$, we trivially have $\left\|Q S^{m p} S^{* m q}\left(\xi_{i}+\xi_{i+N}\right)\right\| \leqslant \sqrt{2}$. For $i \geqslant \alpha(p+q)$, let $j=p-q$, and observe that

$$
S^{m p} S^{* m q} \xi_{i}=S^{m j} \xi_{i}=U_{i} A_{i} f_{j} \quad \text { and } \quad S^{m p} S^{* m q} \xi_{i+N}=S^{m j} \xi_{i+N}=U_{i}^{\prime} A_{i}^{\prime} f_{j} .
$$

Moreover,

$$
\begin{aligned}
\left(U_{i}+U_{i}^{\prime}\right) A_{i} f_{j} & =\left(U_{i} A_{i} f_{j}+U_{i}^{\prime} A_{i}^{\prime} f_{j}\right)+U_{i}^{\prime}\left(A_{i}-A_{i}^{\prime}\right) f_{j} \\
& =S^{m p} S^{* m q}\left(\xi_{i}+\xi_{i+N}\right)+U_{i}^{\prime}\left(A_{i}-A_{i}^{\prime}\right) f_{j} \in G=\operatorname{ker} Q .
\end{aligned}
$$

Hence, $Q S^{m p} S^{* m q}\left(\xi_{i}+\xi_{i+N}\right)=-Q U_{i}^{\prime}\left(A_{i}-A_{i}^{\prime}\right) f_{j}$ and

$$
\left\|Q S^{m p} S^{* m q}\left(\xi_{i}+\xi_{i+N}\right)\right\| \leqslant\left\|A_{i}-A_{i}^{\prime}\right\|<\left(\frac{\alpha}{2}\right)^{i} .
$$

Thus, for any $p, q \geqslant 0$,

$$
\begin{aligned}
2\left\|Q S^{m p} S^{* m q} v\right\|_{2}^{2}= & \sum_{i \geqslant \alpha(p+q)}\left\|Q S^{m p} S^{* m q}\left(\xi_{i}+\xi_{i+N}\right)\right\|^{2} \\
& +\sum_{i<\alpha(p+q)}\left\|Q S^{m p} S^{* m q}\left(\xi_{i}+\xi_{i+N}\right)\right\|^{2} .
\end{aligned}
$$

By the above,

$$
\begin{aligned}
\sum_{i<\alpha(p+q)}\left\|Q S^{m p} S^{* m q}\left(\xi_{i}+\xi_{i+N}\right)\right\|^{2} & \leqslant 2|\{i \in \mathbb{N} \cup\{0\} \mid i<\alpha(p+q)\}| \\
& \leqslant \begin{cases}2(1+\alpha(p+q)), & p+q \geqslant 1, \\
0, & p=q=0\end{cases}
\end{aligned}
$$

and

$$
\sum_{i \geqslant \alpha(p+q)}\left\|Q S^{m p} S^{* m q}\left(\xi_{i}+\xi_{i+N}\right)\right\|^{2}<\sum_{i=\lceil\alpha(p+q)\rceil}^{\infty}\left(\frac{\alpha}{2}\right)^{i}<\alpha
$$


Therefore,

$$
2\left\|Q S^{m p} S^{* m q} v\right\|_{2}^{2} \leqslant 2(1+2 \alpha(p+q)) \leqslant 2 \mathrm{e}^{2 \alpha(p+q)} \leqslant 2 C^{p+q}
$$

for any $p, q \geqslant 0$. Thus, the sequence $\left(\xi_{i}\right)$ is 1 -nice.

Remark 4.6. The proof of Lemma 4.5 actually yields a stronger result: suppose that $\left(\xi_{i}\right)$ is a regular sequence. Then for any $\left(\eta_{k}\right)_{k=1}^{L} \subset X, M \in \mathbb{N}$ and $\varepsilon>0$, there exist $N_{0}^{\prime}\left(\left(\eta_{k}\right)_{k=1}^{L}, \varepsilon, M\right)$ such that for any $N \geqslant N_{0}^{\prime}\left(\left(\eta_{k}\right)_{k=1}^{L}, \varepsilon, M\right)$ and for $\lambda \in \mathbb{C}$ with $|\lambda|=1$ there exists an orthogonal projection $Q_{\lambda}$ for which $\left\|Q_{\lambda} \eta_{k}\right\|>\left(\left\|\eta_{k}\right\|-\varepsilon\right) / \sqrt{2}$ whenever $1 \leqslant k \leqslant L$ and $\left\|Q_{\lambda} S^{m p} S^{* m q} v_{\lambda}\right\|_{2} \leqslant C^{p+q}$. Here, the operator $v_{\lambda}: \ell_{2}^{M} \rightarrow \operatorname{span}\left[\xi_{i} \mid i \in \mathbb{N}\right]$ is defined by setting $v_{\lambda} e_{i}=\left(\xi_{i}+\lambda \xi_{i+N}\right) / \sqrt{2}$. Indeed, in the notation of the lemma, we let $Q_{\lambda}$ be the projection with the kernel span $\left[\left(U_{i}+\lambda U_{i}^{\prime}\right) A_{i} f_{j}|1 \leqslant i \leqslant M| j \mid, \leqslant K_{i}\right]$. The proof of Lemma 4.5 shows that $Q_{1}$ satisfies the above conditions. The case of a general $\lambda$ is dealt with in the same manner.

Proof of Theorem 4.1. By Lemma 4.4, every infinite subspace $Y$ of $X$ contains a regular sequence $\left(\xi_{i}\right)_{i \in \mathbb{N}}$. Let $Z=\operatorname{span}\left[\xi_{i} \mid i \in \mathbb{N}\right]$, and consider $T \in \operatorname{CB}(Z, Y)$ satisfying $\|T\|_{\mathrm{cb}}<\frac{1}{5}$. By Theorem $1.1(\mathrm{v})$, there exists $a \in \ell_{1}\left(C^{|j|}, \mathbb{Z}\right)$ such that $W=T-\tilde{T}_{a}$ is compact, and $\|a\|_{w}<\frac{4}{5}$. Consequently, $\|W\|_{\mathrm{cb}}<1$. By Lemmas 4.3 and $4.5,\|W\|_{2}<4 \sqrt{2}$.

Now consider $T_{1} \in \mathrm{CB}(Z)$, with $\left\|T_{1}\right\|_{\mathrm{cb}}<\frac{1}{85}$. By Lemma 3.6, there exists a diagonal operator $T \in \mathrm{CB}(Z)$ (that is, $T \xi_{i}=\lambda_{i} \xi_{i}$ for any $i \in \mathbb{N}$ ) such that $\|T\|_{\mathrm{cb}}<\frac{1}{5}$ and $\left\|T-T_{1}\right\|_{2}<\frac{16}{85}$. As above, there exists $a=\left(a_{j}\right)_{j \in \mathbb{Z}} \in \ell_{1}\left(C^{|j|}, \mathbb{Z}\right)$ such that $\|a\|_{w}<1$ and $\|W\|_{2}<4 \sqrt{2}$, where $W=T-\left.\tilde{T}_{a}\right|_{z}$. Let $c=\sum_{j \in \mathbb{Z}} a_{j} \alpha_{j}$, where (as in the definition of regularity) $\alpha_{j}=\lim _{i}\left\langle S^{m j} \xi_{i}, \xi_{i}\right\rangle$. Clearly, the sum above converges, and $|c| \leqslant\|a\|_{w}<1$. We shall show that $c I_{Z}-\left.\tilde{T}_{a}\right|_{Z}$ is a Hilbert-Schmidt operator. Indeed, for any $i$,

$$
\lambda_{i}=\left\langle T \xi_{i}, \xi_{i}\right\rangle=\sum_{j \in \mathbb{Z}} a_{j}\left\langle S^{m j} \xi_{i}, \xi_{i}\right\rangle+c_{i},
$$

where $c_{i}=\left\langle W \xi_{i}, \xi_{i}\right\rangle$. However,

$$
\left|\left\langle S^{m j} \xi_{i}, \xi_{i}\right\rangle-\alpha_{j}\right|<\frac{\alpha^{2 i}}{2^{2 i+4}(1+i / \alpha)^{4}}
$$

for $|j| \leqslant K_{i}$, while for other values of $j$ we have $\left|\left\langle S^{m j} \xi_{i}, \xi_{i}\right\rangle-\alpha_{i}\right| \leqslant 2$. Therefore,

$$
\begin{aligned}
\left|\lambda_{i}-c\right| & \leqslant \sum_{j \in \mathbb{Z}}\left|a_{j}\right|\left|\left\langle S^{m j} \xi_{i}, \xi_{i}\right\rangle-\alpha_{j}\right|+\left|c_{i}\right| \\
& \leqslant \sum_{j=-K_{i}}^{K_{i}}\left|a_{j}\right| \frac{\alpha^{2 i}}{2^{2 i+4}(1+i / \alpha)^{4}}+2 \sum_{|j|>K_{i}}\left|a_{j}\right|+\left|c_{i}\right| .
\end{aligned}
$$

However, $\sum_{j \in \mathbb{Z}} C^{|j|}\left|a_{j}\right| \leqslant 1$, and hence $\sum_{|j|>K_{i}}\left|a_{j}\right| \leqslant C^{-\left(K_{i}+1\right)} \leqslant C^{-i / \alpha}$. On the other hand, $\sum_{j=-K_{i}}^{K_{i}}\left|a_{j}\right| \leqslant 1$. Hence, by definition of $\alpha$,

$$
\left|\lambda_{i}-c\right| \leqslant \frac{\alpha^{2 i}}{2^{2 i+4}(1+i / \alpha)^{4}}+2 C^{-i / \alpha}+\left|c_{i}\right| \leqslant \frac{1}{2^{4 i+4}}+2 \mathrm{e}^{-2 i}+\left|c_{i}\right|<2.1 \mathrm{e}^{-2 i}+\left|c_{i}\right| .
$$


Therefore,

$$
\left\|T-c I_{Z}\right\|_{2}^{2}=\sum_{i=1}^{n}\left|\lambda_{i}-c\right|^{2} \leqslant \sum_{i=1}^{n}\left(2.1 \mathrm{e}^{-2 i}+\left|c_{i}\right|\right)^{2}<2 \sum_{i=1}^{n}\left(4.5 \mathrm{e}^{-4 i}+\left|c_{i}\right|^{2}\right) .
$$

We have $\sum_{i}\left|c_{i}\right|^{2} \leqslant\|W\|_{2}^{2}<32$, and hence $\left\|T-c I_{Z}\right\|_{2}<\sqrt{64+9 /\left(\mathrm{e}^{4}-1\right)}$, and

$$
\left\|T_{1}-c I_{Z}\right\|_{2} \leqslant\left\|T-c I_{Z}\right\|_{2}+\left\|T_{1}-T\right\|_{2} \leqslant \sqrt{64+\frac{9}{\mathrm{e}^{4}-1}}+\frac{16}{85}<\frac{700}{85} .
$$

Finally, $|c|=\lim \sup \left|\left\langle T_{1} \xi_{i}, \xi_{i}\right\rangle\right| \leqslant\left\|T_{1}\right\|$.

We next modify our proof to show that $Y$ contains uncountably many subspaces $Z$ with the above properties. For each infinite $\mathcal{F} \subset \mathbb{N}$, let $Z_{\mathcal{F}}=\operatorname{span}\left[\xi_{i} \mid i \in \mathcal{F}\right]$. Any subsequence of a regular sequence is regular, and hence $\operatorname{CB}\left(Z_{\mathcal{F}}, X\right)$ and $\mathrm{CB}\left(Z_{\mathcal{F}}\right)$ are as in the statement of the theorem.

It is well known (see, for example, $[\mathbf{2 1}]$ ) that there exists an uncountable family $\mathcal{I}$ of subsets of $\mathbb{N}$ such that $\mathcal{F} \Delta \mathcal{G}$ is infinite if $\mathcal{F}$ and $\mathcal{G}$ are distinct members of $\mathcal{I}$. We shall show that $Z_{\mathcal{F}}$ and $Z_{\mathcal{G}}$ are not completely isomorphic to each other. Indeed, suppose positive integers $p_{1}<p_{2}<\cdots$ are such that $\left\{p_{1}, p_{2}, \ldots\right\}=\mathcal{F} \backslash \mathcal{G}$. We shall show that no $T \in \mathrm{CB}\left(Z_{\mathcal{F}}, Z_{\mathcal{G}}\right)$ has a bounded inverse.

Indeed, write $T=\sum_{j \in \mathbb{Z}} a_{j} S^{m j}+W$, where $\sum_{j \in \mathbb{Z}} C^{|j|}\left|a_{j}\right|<\infty$ and $W \in \mathcal{S}_{2}$. Let $P$ be the orthogonal projection onto $Z_{\mathcal{G}}$. For each $i$, we have

$$
T \xi_{p_{i}}=P T \xi_{p_{i}}=\sum_{j \in \mathbb{Z}} a_{j} P S^{m j} \xi_{p_{i}}+P W \xi_{p_{i}} .
$$

The sequence $\left(\xi_{i}\right)$ is shift lacunary. Hence, $S^{m j} \xi_{p_{i}}$ is orthogonal to $\xi_{k}$ whenever $p_{i} \neq k$, and $|j| \leqslant K_{p_{i}}$. In this case, $S^{m j} \xi_{p_{i}}$ is orthogonal to $Z_{\mathcal{G}}$ or, in other words, $P S^{m j} \xi_{p_{i}}=0$. As $K_{p_{i}} \geqslant i$ for $i>i_{0}$, we have

$$
\lim _{i}\left\|T \xi_{p_{i}}\right\| \leqslant \lim _{i}\left(\sum_{|j|>i}\left|a_{j}\right|+\left\|W \xi_{p_{i}}\right\|\right)=0
$$

(here we use the compactness of $W$ ). Thus, $T$ is not invertible.

Remark 4.7. In fact, we have shown that the spaces $Z_{\mathcal{F}}$ and $Z_{\mathcal{G}}$ are not semiisomorphic (in the sense of $[\mathbf{2 2}]$ ).

Coordinate subspaces of $X$ give rise to a class of $c$-nice sequences. We say that an increasing sequence of positive integers $\left(n_{i}\right)$ is self-repeating if, for any $N_{0} \in \mathbb{N}$, there exist $N, K>N_{0}$ such that $n_{i+N}=n_{i}+K$ whenever $1 \leqslant i \leqslant N_{0}$.

Lemma 4.8. Suppose an increasing sequence $\left(n_{i}\right)$ is self-repeating. Then the sequence of coordinate vectors $\left(\delta_{n_{i}}\right)_{i \in \mathbb{N}}$ is $\gamma_{1}$-nice, where

$$
\gamma_{1}=\gamma_{1}(C, m)=\max \left\{1, \sup _{j \in \mathbb{N}} \frac{\sqrt{m j}}{\sqrt{2} C^{j}}\right\} \leqslant \max \left\{1, \frac{\sqrt{m}}{2 \sqrt{e \ln C}}\right\} .
$$


Proof. Suppose that $\eta \in X$ and $\operatorname{supp} \eta_{k} \in\left[1, N_{1}\right]$ for $1 \leqslant k \leqslant L$. Find $N_{2}$ such that $C^{N_{2}}>\sqrt{M}$. Find $N, K>\max \left\{n_{M}+m N_{2}, N_{1}\right\}$ so that $n_{i+K}=n_{i}+N$ for $1 \leqslant i \leqslant M$. Let $Q$ be the orthogonal projection with the kernel $G=\operatorname{span}\left[\delta_{k}+\delta_{k+K} \mid 1 \leqslant k \leqslant N\right]$. As in the proof of Lemma 4.5 , we conclude that $\left\|Q \eta_{k}\right\| \geqslant\left\|\eta_{k}\right\| / \sqrt{2}$ for $1 \leqslant k \leqslant L$.

It remains to estimate $\left\|Q S^{m p} S^{* m q} v\right\|_{2}$. If $p+q>N_{2}$, then

$$
\left\|Q S^{m p} S^{* m q} v\right\|_{2} \leqslant\|v\|_{2}=\sqrt{M} \leqslant C^{N_{2}} \leqslant \gamma_{1} C^{p+q} .
$$

If $p+q<N_{2}$, use the equality

$$
2\left\|Q S^{m p} S^{* m q} v\right\|_{2}^{2}=2 \sum_{i=1}^{M}\left\|Q S^{m p} S^{* m q} v e_{i}\right\|^{2}=\sum_{i=1}^{M}\left\|Q S^{m p} S^{* m q}\left(\delta_{n_{i}}+\delta_{n_{i+N}}\right)\right\|^{2} .
$$

If $m q \geqslant n_{i}$, then $S^{* m q} \delta_{n_{i}}=0$, and hence $\left\|Q S^{m p} S^{* m q}\left(\delta_{n_{i}}+\delta_{n_{i+N}}\right)\right\| \leqslant 1$. Otherwise,

$$
S^{m p} S^{* m q}\left(\delta_{n_{i}}+\delta_{n_{i+N}}\right)=\delta_{n_{i}+m(p-q)}+\delta_{n_{i+N}+m(p-q)} \in G,
$$

and $Q S^{m p} S^{* m q}\left(\delta_{n_{i}}+\delta_{n_{i+N}}\right)=0$. Therefore,

$$
2\left\|Q S^{m p} S^{* m q} v\right\|_{2}^{2} \leqslant\left|\left\{i \in \mathbb{N} \mid m q \geqslant n_{i}\right\}\right| \leqslant m q \leqslant m(p+q) .
$$

By our choice of $\gamma_{1},\left\|Q S^{m p} S^{* m q} v\right\|_{2} \leqslant \sqrt{\frac{1}{2} m(p+q)} \leqslant \gamma_{1} C^{p+q}$.

Proof of Theorem 1.1(i), (ii). Consider $T \in \mathrm{CB}(X)$. By the proof of Theorem 1.1 (ii), and by Remark 3.9, there exists a unique $a \in \ell_{1}\left(C^{|j|}, \mathbb{Z}\right)$ such that $\|a\|_{w} \leqslant$ $4\|T\|_{\mathrm{cb}}$, and $T-\tilde{T}_{a}$ is compact. By Lemma 4.8 (applied to the sequence $n_{i}=i$ ), we conclude that $\left\|T-\tilde{T}_{a}\right\|_{2} \leqslant 4 \sqrt{2} \gamma_{1}\left\|T-\tilde{T}_{a}\right\|_{\mathrm{cb}} \leqslant 20 \sqrt{2} \gamma_{1}\|T\|_{\mathrm{cb}}$.

Remark 4.9. Observe that $\mathbb{N}$ contains uncountably many self-repeating sequences. To see this, call an infinite subset of $\mathbb{N}$ self-repeating if the corresponding sequence is selfrepeating. Suppose that $\mathcal{F}$ is a subset of $\mathcal{B}=\left\{10^{n} \mid n \in \mathbb{N}\right\}$. Let $\mathcal{F}_{1}=\mathcal{F} \cup(\mathcal{F} \cap[1,10]+30)$ and, for $n \geqslant 2, \mathcal{F}_{n+1}=\mathcal{F}_{n} \cup\left(\mathcal{F}_{n} \cap\left[1,10^{n}\right]+3 \cdot 10^{n}\right)$. Then the set $\mathcal{F}^{\prime}=\bigcup_{n \in \mathbb{N}} \mathcal{F}_{n}$ is self-repeating (indeed, $\mathcal{F}^{\prime} \cap\left[1,10^{n}\right]+3 \cdot 10^{n}=\mathcal{F}^{\prime} \cap\left[1+3 \cdot 10^{n}, 4 \cdot 10^{n}\right]$ for every $n \in \mathbb{N}$ ), and $\mathcal{F}^{\prime} \cap \mathcal{B}=\mathcal{F}$. Thus, $\mathcal{F} \mapsto \mathcal{F}^{\prime}$ is an injection from $2^{\mathcal{B}}$ to the family of self-repeating sets.

For a self-repeating set $\mathcal{F}$, let $Y_{\mathcal{F}}=\operatorname{span}\left[\delta_{i} \mid i \in \mathbb{N}\right]$. If $\mathcal{F}$ and $\mathcal{G}$ are subsets of $\mathcal{B}$, and $|\mathcal{F} \Delta \mathcal{G}|=\infty$, then, arguing as in the last part of Theorem 4.1, we conclude that $Y_{\mathcal{F}^{\prime}}$ and $Y_{\mathcal{G}^{\prime}}$ are not completely isomorphic. Thus, $X$ contains an uncountable family of coordinate subspaces that are not completely isomorphic to each other.

\section{Failure of the OAP}

Proof of Theorem 1.1 (iii). Suppose that $Y$ is an infinite-dimensional subspace $Y$ of $X$. By Lemma 4.4, $Y$ contains a regular sequence $\left(\xi_{i}\right)$. Select $N \geqslant 21$. For every 
$n \geqslant N$ and $s= \pm 1$ there exists $r=r(n, s)$ such that $n_{r}=\operatorname{dim} E_{r}=2^{n+1}$, and $\left\|u_{r}-w_{n s}\right\|_{1}<2^{-(n+1)}$, where $w_{n s}: X \rightarrow E_{r}$ is defined by

$$
w_{n s} \xi=2^{-(n+1) / 2} \sum_{i=1}^{2^{n}}\left\langle\xi, \xi_{i}+s \xi_{i+2^{n}}\right\rangle e_{i} \quad \text { for any } \xi \in X
$$

(here $\left(e_{i}\right)_{i=1}^{2^{n}}$ is the canonical basis for $E_{r}$ ). Define $v_{n s}: E_{r}^{*} \rightarrow Z$ by letting $v_{n s} e_{i}=$ $\left(\xi_{i}-s \xi_{i+2^{n}}\right) / \sqrt{2}$. We show first that $\left\|v_{n s}\right\|_{\mathrm{cb}} \leqslant 1$. Indeed, by (2.1) (see the proof of Lemma 2.2),

$$
\begin{aligned}
\left\|v_{n s}\right\|_{\mathrm{cb}} & \leqslant \max \left\{1, \sup _{p, q \geqslant 0} C^{-(p+q)}\left\|u_{r} S^{m p} S^{* m q} v_{n s}\right\|_{1}\right\} \\
& \leqslant \max \left\{1, \sup _{p, q \geqslant 0} C^{-(p+q)}\left(\left\|w_{n s} S^{m p} S^{* m q} v_{n s}\right\|_{1}+\left\|u_{r}-w_{n s}\right\|_{1}\right)\right\} .
\end{aligned}
$$

However,

$$
\begin{aligned}
2\left\|w_{n s} S^{m p} S^{* m q} v_{n s}\right\|_{1} & \leqslant 2 \sum_{k, \ell=1}^{2^{n}}\left|\left\langle w_{n s} S^{m p} S^{* m q} v_{n s} e_{k}, e_{\ell}\right\rangle\right| \\
& =\sum_{k, \ell=1}^{2^{n}}\left|\left\langle\left(\xi_{k}+s \xi_{k+2^{n}}\right), S^{m p} S^{* m q}\left(\xi_{\ell}-s \xi_{\ell+2^{n}}\right)\right\rangle\right| v \\
& \leqslant \sum_{i=1}^{2^{n}}\left|\left\langle\xi_{i}, S^{m p} S^{* m q} \xi_{i}\right\rangle-\left\langle\xi_{i+2^{n}}, S^{m p} S^{* m q} \xi_{i+2^{n}}\right\rangle\right| \\
& +\sum_{k \neq \ell, 1 \leqslant k, \ell \leqslant 2^{n+1}}\left|\left\langle\xi_{k}, S^{m p} S^{* m q} \xi_{\ell}\right\rangle\right| .
\end{aligned}
$$

Using the definition of regularity as in the proof of Lemma 4.5, and recalling that $\alpha \leqslant \frac{1}{2}$, we conclude that

$$
\left|\left\langle\xi_{i}, S^{m p} S^{* m q} \xi_{i}\right\rangle-\left\langle\xi_{i+2^{n}}, S^{m p} S^{* m q} \xi_{i+2^{n}}\right\rangle\right| \leqslant \frac{\alpha^{2 i}}{2^{2 i+3}(1+i / \alpha)^{4}}<\frac{1}{2^{4 i+3}}
$$

if $\max \{p, q\} \leqslant 2(1+i / \alpha)$. Thus,

$$
\begin{aligned}
\sum_{i=1}^{2^{n}} \mid\left\langle\xi_{i}, S^{m p} S^{* m q} \xi_{i}\right\rangle & -\left\langle\xi_{i+2^{n}}, S^{m p} S^{* m q} \xi_{i+2^{n}}\right\rangle \mid \\
\leqslant & \sum_{i=1}^{\lfloor\alpha(p+q) / 2\rfloor}\left(\left|\left\langle\xi_{i}, S^{m p} S^{* m q} \xi_{i}\right\rangle\right|+\left|\left\langle\xi_{i+2^{n}}, S^{m p} S^{* m q} \xi_{i+2^{n}}\right\rangle\right|\right) \\
& +\sum_{i=\lfloor\alpha(p+q) / 2\rfloor+1}^{2^{n}}\left|\left\langle\xi_{i}, S^{m p} S^{* m q} \xi_{i}\right\rangle-\left\langle\xi_{i+2^{n}}, S^{m p} S^{* m q} \xi_{i+2^{n}}\right\rangle\right| \\
\leqslant & \alpha(p+q)+\sum_{i=1}^{2^{n}} \frac{1}{2^{4 i+3}}<\alpha(p+q)+\frac{1}{100} .
\end{aligned}
$$


On the other hand, by $(3.1),\left\langle\xi_{k}, S^{m p} S^{* m q} \xi_{\ell}\right\rangle=0$ if $p+q \leqslant \max \{k, \ell\} / \alpha$, and hence

$\sum_{k \neq \ell, 1 \leqslant k, \ell \leqslant 2^{n+1}}\left|\left\langle\xi_{k}, S^{m p} S^{* m q} \xi_{\ell}\right\rangle\right| \leqslant|\{(k, \ell) \mid k \neq \ell, \max \{k, \ell\}<\alpha(p+q)\}|<(\alpha(p+q))^{2}$.

Thus, by (5.2),

$$
\left\|w_{n s} S^{m p} S^{* m q} v_{n s}\right\|_{1} \leqslant \frac{1}{2}\left(\frac{1}{100}+\alpha(p+q)+(\alpha(p+q))^{2}\right)
$$

and therefore, by (5.1),

$$
\left\|v_{n s}\right\|_{\mathrm{cb}} \leqslant \max \left\{1, \sup _{p, q \geqslant 0} \frac{1}{2} C^{-(p+q)}\left(1+\alpha(p+q)+(\alpha(p+q))^{2}\right)\right\}=1,
$$

since $1+\alpha(p+q)+(\alpha(p+q))^{2} \leqslant(1+\alpha(p+q))^{2} \leqslant \mathrm{e}^{2 \alpha(p+q)} \leqslant C^{(p+q)}$.

If $Y$ has the OAP, there exists, by [21, Lemma 6.1], a finite-rank map $T: Z \rightarrow Y$ such that $T \xi_{i}=\xi_{i}$ for $1 \leqslant i \leqslant 2^{N}$ and $\left\|T v_{n s}\right\|_{\mathrm{cb}} \leqslant 2^{n / 4-4}$ for any $n \geqslant N$ and $s= \pm 1$. By the above, $\left\|v_{n s}\right\|_{\mathrm{cb}} \leqslant 1$ and, moreover, $\left\|w_{n s}\right\|_{\mathrm{cb}} \leqslant\left\|u_{r}-w_{n s}\right\|_{1}+\left\|u_{r}\right\|_{\mathrm{cb}}<\frac{8}{7}$. Thus,

$$
\left\|w_{n s} T v_{n s}\right\|_{1} \leqslant 5\left\|w_{n s} T v_{n s}\right\|_{\mathrm{cb}}<7\left\|T v_{n s}\right\|_{\mathrm{cb}}<2^{n / 4-1}
$$

and therefore

$$
\begin{aligned}
& 2^{n / 2+1} \sum_{i=1}^{2^{n}}\left|\left\langle w_{n s} T v_{n s} e_{i}, e_{i}\right\rangle\right| \\
& \quad=\sum_{i=1}^{2^{n}}\left|\left\langle T \xi_{i}, \xi_{i}\right\rangle-\left\langle T \xi_{i+2^{n}}, \xi_{i+2^{n}}\right\rangle+s\left(\left\langle T \xi_{i}, \xi_{i+2^{n}}\right\rangle-\left\langle T \xi_{i+2^{n}}, \xi_{i}\right\rangle\right)\right| \\
& \leqslant 2^{n / 2+1}\left\|w_{n s} T v_{n s}\right\|_{1} \leqslant 2^{3 n / 4} .
\end{aligned}
$$

Averaging over $s$, we obtain

$$
\begin{aligned}
2^{n / 2} \sum_{i=1}^{2^{n}} \mid\left\langle w_{n,+1} T v_{n,+1} e_{i}, e_{i}\right\rangle+\left\langle w_{n,-1}\right. & \left.T v_{n,-1} e_{i}, e_{i}\right\rangle \mid \\
& =\sum_{i=1}^{2^{n}}\left|\left\langle T \xi_{i}, \xi_{i}\right\rangle-\left\langle T \xi_{i+2^{n}}, \xi_{i+2^{n}}\right\rangle\right| \leqslant 2^{3 n / 4} .
\end{aligned}
$$

Let $A_{n}=\operatorname{Re} \sum_{i=1}^{2^{n}}\left\langle T \xi_{i}, \xi_{i}\right\rangle$. Then $A_{N}=2^{N}$ and, by the triangle inequality, $A_{n+1} \geqslant$ $2 A_{n}-2^{3 n / 4}$. It is easy to show by induction that $A_{n}>3 \cdot 2^{n-2}+7 \cdot 2^{3 n / 4}$ for any $n \geqslant N$. On the other hand, $\sup _{n} A_{n} \leqslant\|T\|_{1}<\infty$ : a contradiction.

\section{6. $K$-theory of $\mathrm{CB}(X(C, m))$}

In this section, we prove Theorem 1.1 (iv). First, recall the basics. Suppose that $\mathcal{A}$ is a Banach algebra. We denote by $M_{n}(\mathcal{A})$ the algebra of $n \times n$ matrices with entries from $\mathcal{A}$, with obvious multiplication. For $a_{i} \in M_{n_{i}}(\mathcal{A}), i=1,2$, we denote by $a_{1} \oplus a_{2}$ the element

$$
\left(\begin{array}{cc}
a_{1} & 0 \\
0 & a_{2}
\end{array}\right) \in M_{n_{1}+n_{2}}(\mathcal{A}) .
$$


To define the $K$-groups, first assume that $\mathcal{A}$ has identity $I$. For $n \in \mathbb{N}$, $\operatorname{IP}_{n}(\mathcal{A})$ denotes the set of idempotents (or projections) in $M_{n}(\mathcal{A})$. For $k<n$, we view $\operatorname{IP}_{k}(\mathcal{A})$ as a subset of $\operatorname{IP}_{n}(\mathcal{A})$, by identifying $p \in \operatorname{IP}_{k}(\mathcal{A})$ with $p \oplus 0^{(n-k)} \in \operatorname{IP}_{n}(\mathcal{A})$ (here and below, $0^{(s)}$ stands for the zero element of $M_{s}(\mathcal{A})$ ). We let $\operatorname{IP}_{\infty}(\mathcal{A})=\bigcup_{n \in \mathbb{N}} \operatorname{IP}_{n}(\mathcal{A})$.

We say that $p, q \in \operatorname{IP}_{n}(\mathcal{A})$ are algebraically equivalent $\left(p \sim_{a} q\right)$ if there exist $u, v \in$ $M_{n}(\mathcal{A})$ satisfying $v u=p, u v=q . p$ and $q$ are 0 -equivalent $\left(p \sim_{0} q\right)$ if $p \oplus I^{(k)} \sim_{a} q \oplus I^{(k)}$ for some $k \geqslant 0$ (here $I^{(k)}$ is the element of $M_{k}(\mathcal{A})$ consisting of $k$ copies of $I$ on the diagonal and zeros elsewhere). Equivalently, $p \sim_{0} q$ if $p \oplus r \sim_{a} q \oplus r$ for some $r \in \operatorname{IP}_{\infty}(\mathcal{A})$.

The 0 -equivalence class of $p \in \operatorname{IP}_{\infty}(\mathcal{A})$ is denoted by $[p]_{0}$. Following $[3, \S 5]$, we introduce the (abelian) group $K_{0}(\mathcal{A})$ as the set of all differences $[p]_{0}-[q]_{0}$, with $p, q \in \operatorname{IP}_{\infty}(A)$. The equality $[p]_{0}-[q]_{0}=\left[p^{\prime}\right]_{0}-\left[q^{\prime}\right]_{0}$ holds if $p \oplus q^{\prime} \sim_{0} p^{\prime} \oplus q$. Addition is defined by $[p]_{0}+[q]_{0}=[p \oplus q]_{0}$, and $[0]_{0}$ serves as the neutral element of this group.

To define the group $K_{1}(\mathcal{A})$, consider the set $\mathrm{GL}_{n}(\mathcal{A})$ of all invertible elements of $M_{n}(\mathcal{A})$. For $\ell<n$, identify $U \in \mathrm{GL}_{\ell}(\mathcal{A})$ with $U \oplus I^{(n-\ell)} \in \mathrm{GL}_{n}(\mathcal{A})$. Let $\mathrm{GL}_{\infty}(\mathcal{A})=$ $\bigcup_{n} \mathrm{GL}_{n}(\mathcal{A})$. We say that $U \in \mathrm{GL}_{\ell}(\mathcal{A})$ and $V \in \mathrm{GL}_{n}(\mathcal{A})$ are homotopically equivalent $\left(U \sim_{1} V\right.$, or $\left.U \sim_{h} V\right)$ if, for some $k \geqslant \max \{\ell, n\}$, there exists a continuous path $h:[0,1] \rightarrow \mathrm{GL}_{k}(\mathcal{A})$ such that $h(0)=U \oplus I^{(k-\ell)}$ and $h(1)=V \oplus I^{(k-n)}$. $[U]_{1}$ denotes the equivalence class of $U$. The abelian group $K_{1}(\mathcal{A})$ is generated by the set of equivalence classes $\mathrm{GL}_{\infty}(\mathcal{A}) / \sim_{1}$, equipped with the addition $[U]_{1}+[V]_{1}=[U \oplus V]_{1}$ and with the neutral element $[I]_{1}$.

If $\mathcal{A}$ is a non-unital Banach algebra, denote by $\mathcal{A}^{\sharp}$ its unitization, with the identity $I$. $s: \mathcal{A}^{\sharp} \rightarrow \mathbb{C}$ denotes the scalar map: $s(a+\lambda I)=\lambda$, for any $a \in \mathcal{A}$ and $\lambda \in \mathbb{C}$. $\boldsymbol{s}_{n}$ stands for $I_{M_{n}} \otimes s$ (viewed as a map from $M_{n}\left(\mathcal{A}^{\sharp}\right)$ to $\left.M_{n}\right)$. Clearly, if $a \in M_{n}\left(\mathcal{A}^{\sharp}\right)$ is an idempotent (or invertible), then so is $\boldsymbol{s}_{n}(a) \in M_{n}$. The $K$-groups of $\mathcal{A}$ can be described as follows: $K_{1}(\mathcal{A})=K_{1}\left(\mathcal{A}^{\sharp}\right)$, and $K_{0}(\mathcal{A})$ is the subgroup of $K_{0}\left(\mathcal{A}^{\sharp}\right)$ generated by $[p]_{0}-\left[\boldsymbol{s}_{n}(p)\right]_{0}$, for $p \in \operatorname{IP}_{n}\left(\mathcal{A}^{\sharp}\right)$.

Now suppose that $\pi$ is a bounded algebraic homomorphism between Banach algebras $\mathcal{A}$ and $\mathcal{B}$. Clearly, $I_{M_{n}} \otimes \pi$ maps idempotents (and, in the case of unital algebras, invertible elements) of $M_{n}(\mathcal{A})$ into idempotents (respectively, invertible elements) of $M_{n}(\mathcal{B})$. Attaching the unit if necessary, we define the group homomorphisms $K_{\ell}(\pi): K_{\ell}(\mathcal{A}) \rightarrow$ $K_{\ell}(\mathcal{B}):[a]_{\ell} \mapsto\left[I_{M_{n}} \otimes \pi(a)\right]_{\ell}$ for $\ell=0,1$ and appropriate $a \in M_{n}(\mathcal{A})$.

The reader is referred to $[\mathbf{3}, \mathbf{1 3}]$ for more information on the $K$-theory for Banach algebras. Applications of $K$-theory to algebras of bounded operators can be found in $[\mathbf{1 1}, \mathbf{1 3}, \mathbf{2 8}]$. We quote some specific results later on.

By Theorem 1.1(i), we have a short exact sequence

$$
0 \rightarrow \mathcal{S}_{2} \stackrel{i}{\rightarrow} \mathrm{CB}(X) \stackrel{\phi}{\rightarrow} \mathcal{Y} \rightarrow 0
$$

where $\mathcal{Y}$ is the algebra of analytic functions on the annulus $\mathbb{A}$ with coefficients in $\ell_{1}\left(C^{|j|}, \mathbb{Z}\right), i$ is the natural injection and $\phi$ is the quotient map. In fact, $\phi$ was described explicitly in the paragraphs following Remark 3.9. There, we observed that for any $T \in$ $\mathrm{CB}(X)$ there exist unique $a=\left(a_{j}\right)_{j \in \mathbb{Z}} \in \ell_{1}\left(C^{|j|}, \mathbb{Z}\right)$ and $W \in \mathcal{S}_{2}$ for which $T=\tilde{T}_{a}+W$. We defined the function $\phi(T)$ by setting, for $z \in \mathbb{A}, \phi(T)(z)=\sum_{j \in \mathbb{Z}} a_{j} z^{j}$. Moreover, it was observed that $\phi$ is an algebraic isomorphism, that is, $\phi\left(T_{1} T_{2}\right)=\phi\left(T_{1}\right) \phi\left(T_{2}\right)$. 
We first calculate the $K$-groups of the algebras $\mathcal{S}_{2}$ and $\mathcal{Y}$, and then use Bott periodicity [3, Chapter 9] to prove Theorem 1.1 (iv).

To deal with $\mathcal{Y}$, observe that idempotents of $M_{n}(\mathcal{Y})$ are precisely those functions $f: \mathbb{A} \rightarrow M_{n}$ whose entries belong to $\mathcal{Y}$ and such that $f(z)$ is a projection (not necessarily self-adjoint) for any $z \in \mathbb{A}$. For $f \in \operatorname{IP}_{n}(\mathcal{Y})$ define $\operatorname{rank} f=\operatorname{rank} f(z)$ (clearly, the latter does not depend on the choice of $z \in \mathbb{A}$ ).

To classify the equivalence classes of $f \in \mathrm{GL}_{n}(\mathcal{Y})$, observe that, since the Banach algebra $\mathcal{Y}$ is commutative, $f \in M_{n}(\mathcal{Y})$ is invertible if and only if $\operatorname{det} f$ is an invertible element of $\mathcal{Y}$. By $[\mathbf{4}$, Theorem 4.6.12], the spectrum of $\mathcal{Y}$ is identified with $\mathbb{A}$ via the evaluation map. Thus, $f \in M_{n}(\mathcal{Y})$ is invertible if and only if $\operatorname{det} f \operatorname{does}$ not vanish anywhere on $\mathbb{A}$.

If $g \in \mathcal{Y}$ does not vanish on $\mathbb{A}$, denote by wind $g$ the winding number of the function $\mathbb{T} \rightarrow \mathbb{C}: z \mapsto g(r z), C^{-1} \leqslant r \leqslant C$, around 0. By continuity, this winding number does not depend on $r$.

\section{Lemma 6.1.}

(a) The group $K_{1}(\mathcal{Y})$ is homomorphic to $\mathbb{Z}$, via $[f]_{1} \mapsto-$ wind $\operatorname{det} f, f \in \mathrm{GL}_{n}(\mathcal{Y})$.

(b) The group $K_{0}(\mathcal{Y})$ is homomorphic to $\mathbb{Z}$, via $[f]_{0} \mapsto \operatorname{rank} f, f \in \operatorname{IP}_{n}(\mathcal{Y})$.

Proof. First we tackle (a). It is well known in $K$-theory that $K_{1}(C(\mathbb{T}))=\mathbb{Z}$, with the group homomorphism given by $[f]_{1} \mapsto-$ wind det $f$ for $f \in \mathrm{GL}_{n}(C(\mathbb{T}))$ (this follows, for instance, from [10, Proposition 8.3.1]). Moreover, the restriction map $j: C(\mathbb{A}) \rightarrow C(\mathbb{T}):\left.f \mapsto f\right|_{\mathbb{T}}$ determines a group homomorphism between $K_{1}(C(\mathbb{A}))$ and $K_{1}(C(\mathbb{T}))$. Indeed, suppose $f \in \mathrm{GL}_{n}(C(\mathbb{A}))$ is such that there exists a continuous map $\Phi:[0,1] \rightarrow \mathrm{GL}_{n}(C(\mathbb{T}))$ such that $\Phi(0)=j f$ and $\Phi(1)=\mathbf{1}_{M_{n}}$ (that is, $\Phi(1)(z)=I_{M_{n}}$ for any $z \in \mathbb{T})$. Define the maps $\Phi_{1}, \Phi_{2}:[0,1] \rightarrow \mathrm{GL}_{n}(C(\mathbb{A}))$, for $t \in[0,1]$ and $z \in \mathbb{A}$, by setting $\Phi_{1}(t)(z)=\Phi(t)(z /|z|)$ and

$$
\Phi_{2}(t)(z)= \begin{cases}f(z), & t C^{-1}+(1-t) \leqslant|z| \leqslant t C+(1-t), \\ f\left(z\left(t C^{-1}+(1-t)\right) /|z|\right), & C^{-1} \leqslant|z|<t C^{-1}+(1-t), \\ f(z(t C+(1-t)) /|z|), & t C+(1-t)<|z| \leqslant C .\end{cases}
$$

Clearly, both $\Phi_{1}$ and $\Phi_{2}$ are continuous, $\Phi_{1}(1)=\mathbf{1}_{M_{n}}$ (as a function on $\left.\mathbb{A}\right), \Phi_{2}(1)=f$ and $\Phi_{1}(0)=\Phi_{2}(0)$.

This shows that $f$ and $g$ lie in the same connected component of $\mathrm{GL}_{n}(C(\mathbb{A}))$ if and only if $j f$ and $j g$ lie in the connected component of $\mathrm{GL}_{n}(C(\mathbb{T}))$. Therefore, the map $K_{1}(C(\mathbb{A})) \rightarrow \mathbb{Z}:[f]_{1} \mapsto-$ wind $\operatorname{det} f$ is a group homomorphism.

Moreover, by [27, Theorem 3.8], the Gelfand transform induces a homomorphism between $K_{1}(\mathcal{Y})$ and $K_{1}(C(\mathbb{A}))$. That is, $f, g \in \mathrm{GL}_{\infty}(\mathcal{Y})$ are connected by a path lying in $\mathrm{GL}_{\infty}(\mathcal{Y})$ if and only if they are connected by a path lying in $\mathrm{GL}_{\infty}(C(\mathbb{A}))$. Conversely, every equivalence class of $\mathrm{GL}_{\infty}(C(\mathbb{A}))$ has a representative in $\mathrm{GL}_{\infty}(\mathcal{Y})$ (in fact, $f: z \mapsto z^{-k}$ can work as such a representative, for an appropriate $k \in \mathbb{Z}$ ). Thus, $K_{1}(\mathcal{Y}) \rightarrow \mathbb{Z}:[f]_{1} \mapsto-$ wind $\operatorname{det} f$ is a group homomorphism. 
Part (b) is proved in a similar fashion. The only additional ingredient is the connection between homotopy equivalence and algebraic equivalence between classes of idempotents. More precisely, suppose $\mathcal{A}$ is a Banach algebra. By [3, Propositions 4.3.1 and 4.4.1], if $p \in \operatorname{IP}_{n}(\mathcal{A})$ and $q \in \operatorname{IP}_{\ell}(\mathcal{A})$ are algebraically equivalent, then, for sufficiently large $k$, there exists a continuous map $h:[0,1] \rightarrow \operatorname{IP}_{k}(\mathcal{A})$ such that $h(0)=p \oplus 0^{(k-n)}$ and $h(1)=q \oplus 0^{(k-m)}$. Conversely, if such a map $h$ exists, then $p \sim_{a} q[\mathbf{3}$, Proposition 4.3.3].

To tackle $\mathcal{S}_{2}$, we need a technical lemma (which may be known to specialists).

Lemma 6.2. If $W$ is a Hilbert-Schmidt map on a Hilbert space $H$, and $I+W$ is invertible, then $(I+W)^{-1}-I$ is Hilbert-Schmidt.

Proof. Let $T=I+W$. Find a subspace $E \hookrightarrow H$ of finite codimension, for which $\left\|\left.W\right|_{E}\right\|<1 / 2$. Let $F=H \ominus E, E^{\prime}=T(E)$, and $F^{\prime}=T(F)$. Clearly, $E^{\prime}$ and $F^{\prime}$ are closed, and $\operatorname{dim} H / E^{\prime}=\operatorname{dim} H / E$ ( $T$ has Fredholm index 0$)$. By the invertibility of $T, E^{\prime} \cap F^{\prime}=$ $\emptyset$, and hence $H=\operatorname{span}\left[E^{\prime}, F^{\prime}\right]$. Let $W_{0}=W P_{E}$, where $P_{E}$ is the orthogonal projection onto $E$. Then $W_{1}=\sum_{k=1}^{\infty}(-1)^{k} W_{0}^{k}$ is Hilbert-Schmidt, and $I+W_{1}=\left(I+W_{0}\right)^{-1}$. Therefore, $\left.T\left(I+W_{1}\right)\right|_{E^{\prime}}=I_{E^{\prime}}$ (the identity map on $E^{\prime}$ ).

Denote by $Q$ the projection onto $E^{\prime}$ along $F^{\prime}$ (that is, $\operatorname{ran} Q=E^{\prime}$ and $\left.\operatorname{ker} Q=F^{\prime}\right)$. Let $W_{2}=W_{1} Q+\left(\left(\left.T\right|_{F}\right)^{-1}-I_{F^{\prime}}\right)(I-Q)$. Clearly, $W_{2}$ is Hilbert-Schmidt, and

$$
T\left(I+W_{2}\right)=T\left(I+W_{2}\right) Q+T\left(I+W_{2}\right)(I-Q)=Q+(I-Q)=I .
$$

Since both $T$ and $I+W_{2}$ are Fredholm operators of index $0, I+W_{2}=T^{-1}$.

Our treatment of the $K$-groups of $\mathcal{S}_{2}$ owes much to [11]. We view the unitization of $\mathcal{S}_{2}$ (denoted by $\mathcal{S}_{2}^{\sharp}$ ) as the subalgebra of $\mathrm{CB}(X)$, generated by $\mathcal{S}_{2}$ and the identity $I$. By definition, $X^{n}$ is isometric to $\ell_{2}^{n}\left(\ell_{2}\right)$, and hence $M_{n}\left(\mathcal{S}_{2}^{\sharp}\right)$ can be viewed as a subalgebra of $\mathrm{CB}\left(X^{n}\right) \subset M_{n}\left(B\left(\ell_{2}\right)\right)$. Moreover, for any $a \in M_{n}\left(\mathcal{S}_{2}^{\sharp}\right), a-s_{n}(a) \otimes I$ is a Hilbert-Schmidt operator on $\ell_{2}^{n}\left(\ell_{2}\right)$.

Corollary 6.3. Suppose that $A$ is an element of $M_{n}\left(\mathcal{S}_{2}^{\sharp}\right)$, invertible in $M_{n}\left(B\left(\ell_{2}\right)\right.$ ). Then its inverse belongs to $M_{n}\left(\mathcal{S}_{2}^{\sharp}\right)$. Furthermore, the spectrum of $A$ is at most countable, with at most finitely many accumulation points.

Proof. If $A$ is invertible, then $a=s_{n}(A)$ is an invertible element of $M_{n}$. Thus, multiplying by $a^{-1} \otimes I$ if necessary, we can assume that $A=I+W$, where $I$ is the identity on $\ell_{2}^{n}\left(\ell_{2}\right)$, and $W \in M_{n}\left(\mathcal{S}_{2}\right)$. By Lemma $6.2, A^{-1} \in M_{n}\left(\mathcal{S}_{2}^{\sharp}\right)$.

By [12, Proposition 2.c.10], $A-\lambda \otimes I$ is a Fredholm operator of index 0 for any $\lambda \notin \sigma\left(\boldsymbol{s}_{n}(A)\right)$. Then, for any $\lambda \in \sigma(A) \backslash \sigma\left(\boldsymbol{s}_{n}(A)\right)$, there exists $\varepsilon>0$ such that $\mu \notin \sigma(A)$ whenever $0<|\lambda-\mu|<\varepsilon$. As in the proof of [12, Lemma 2.c.12], it follows that $\sigma(A)$ is at most countable, and $\sigma\left(\boldsymbol{s}_{n}(A)\right)$ contains all the accumulation points of $\sigma(A)$.

Below, we denote by $Q_{n}, n \geqslant 0$, a rank- $n$ idempotent in $\mathcal{S}_{2}$. Clearly, all such idempotents of the same rank are algebraically equivalent. 


\section{Lemma 6.4.}

(a) The group $K_{1}\left(\mathcal{S}_{2}\right)$ is homomorphic to $\{0\}$.

(b) The group $K_{0}\left(\mathcal{S}_{2}\right)$ can be identified with $\mathbb{Z}$, via $n \mapsto\left[Q_{n}\right]_{0}$ for $n \geqslant 0$ and $n \mapsto$ $-\left[Q_{-n}\right]_{0}$ for $n<0$.

Proof. (a) Suppose that $A$ is an invertible element of $M_{n}\left(\mathcal{S}_{2}^{\sharp}\right)$. By Corollary 6.3, and by [11, Lemma 3.2$]$, we show that $A$ can be connected to the identity by a path of invertible elements.

(b) For any $p \in \operatorname{IP}_{n}\left(\mathcal{S}_{2}^{\sharp}\right), \boldsymbol{s}_{n}(p) \in \operatorname{IP}\left(M_{n}\right)$ is similar to a diagonal $n \times n$ matrix. Hence, one can assume that $p=a \otimes I+W$, where $a \in M_{n}$ is a diagonal projection and $W$ is Hilbert-Schmidt. By the proof of [12, Theorem 2.c.13] (adapted to the operator space setting in [18]), $p$ is algebraically equivalent to $p_{1} \oplus \cdots \oplus p_{n}$, where $p_{1}, \ldots, p_{n}$ are idempotents in $\mathcal{S}_{2}^{\sharp}$. Moreover, if $P \in \operatorname{IP}\left(\mathcal{S}_{2}^{\sharp}\right)$, then $P \sim_{a} s(P) I+(-1)^{s(P)} Q_{n(P)}$, with $\boldsymbol{s}(P) \in\{0,1\}$, and $n(P) \in\{0\} \cup \mathbb{N}$. It is easy to see that $P_{1} \oplus \cdots \oplus P_{\ell} \sim_{a} P_{1}^{\prime} \oplus \cdots \oplus P_{k}^{\prime}$ if and only if

$$
\sum_{j=1}^{\ell} \boldsymbol{s}\left(P_{j}\right)=\sum_{j=1}^{k} \boldsymbol{s}\left(P_{j}^{\prime}\right) \quad \text { and } \quad \sum_{j=1}^{\ell}(-1)^{s\left(P_{j}\right)} n\left(P_{j}\right)=\sum_{j=1}^{k}(-1)^{s\left(P_{j}^{\prime}\right)} n\left(P_{j}^{\prime}\right) .
$$

Therefore, the Banach algebra $\mathcal{S}_{2}^{\sharp}$ has cancellation: if $p_{1}, p_{2} \in \operatorname{IP}_{\infty}\left(\mathcal{S}_{2}^{\sharp}\right)$ satisfy $p_{1} \sim_{0} p_{2}$ (that is, $p_{1} \oplus q \sim_{a} p_{2} \oplus q$ for some $q \in \operatorname{IP}_{\infty}\left(\mathcal{S}_{2}^{\sharp}\right)$ ), then $p_{1} \sim_{a} p_{2}$. We complete the proof as in [11, Theorem 3.9].

Proof of Theorem 1.1 (iv). We rely on the well-known fact (see, for example, [3, Chapter 9]) that (6.1) gives rise to a sequence of group homomorphisms which is 'exact in the middle'. That is, $\operatorname{ker} K_{\ell}(\phi)=\operatorname{ran} K_{\ell}(i)$, where $\ell=0,1$, and the maps $i$ and $\phi$ are as in (6.1).

To prove the theorem for $K_{1}$, note that, if $T$ is an invertible element in $M_{n}(\mathrm{CB}(X))=$ $\mathrm{CB}\left(X^{n}\right)$, then $\operatorname{det} \phi_{n}(T)$ does not vanish on $\mathbb{A}$ (here $\left.\phi_{n}=I_{M_{n}} \otimes \phi\right)$. Write $T=\tilde{T}_{a}+W$, where $a \in M_{n}\left(\ell_{1}\left(C^{|j|}, \mathbb{Z}\right)\right)$ corresponds to $\phi_{n}(T)$, and $W$ is Hilbert-Schmidt. Since $T$ is invertible, $\tilde{T}_{a}$ must have Fredholm index 0 . Thus, by [5, Chapter 1] or [15], wind $\operatorname{det}\left(\phi_{n}(T)\right)=0$, and therefore, by Lemma $6.1, K_{1}(q)\left([T]_{1}\right)=0$. In other words, $K_{1}(\mathrm{CB}(X))=\operatorname{ker} K_{1}(q)$. However, $\operatorname{ker} K_{1}(q)=\operatorname{ran} K_{1}(i)$, by 'exactness in the middle', and $\operatorname{ran} K_{1}(i)=\{0\}$, by Lemma 6.4. Thus, $K_{1}(\mathrm{CB}(X))=\{0\}$.

Next we deal with $K_{0}$. By Lemma $6.1, K_{0}(q)\left([p]_{0}-[q]_{0}\right)=\operatorname{rank} \phi_{n}(p)-\operatorname{rank} \phi_{k}(q)$ for $p \in \mathrm{IP}_{n}(\mathrm{CB}(X))$ and $q \in \mathrm{IP}_{k}(\mathrm{CB}(X))$. Thus, $\operatorname{ran} K_{0}(q)$ can be identified with $\mathbb{Z}$, and $\operatorname{ran} K_{0}(q)=K_{0}(\mathcal{Y})$. Moreover, define a group homomorphism $\mu: \mathbb{Z}=K_{0}(\mathcal{Y}) \rightarrow$ $K_{0}(\mathrm{CB}(X))$ by setting $\mu(n)=\left[I^{(n)}\right]_{0}$ for $n \geqslant 0$ and $\mu(n)=-\left[I^{(n)}\right]_{0}$ for $n<0$. Clearly, $K_{0}(q) \circ \mu$ is the identity on $\mathbb{Z}$. 'Exactness in the middle' then implies that $K_{0}(\mathrm{CB}(X))=$ $\mathbb{Z} \oplus \operatorname{ran} K_{0}(i)$.

It remains to show that $\operatorname{ran} K_{0}(i)=\mathbb{Z} / m \mathbb{Z}$. By Lemma 6.4 , we have a bijective group homomorphism $\nu: \mathbb{Z} \rightarrow K_{0}\left(\mathcal{S}_{2}\right)$, taking $n$ to $\left[Q_{n}\right]_{0}$ for $n \geqslant 0$ and to $-\left[Q_{-n}\right]_{0}$ for $n<0$. 
Thus, we need to prove that $K_{0}(i)\left(\nu\left(n_{1}\right)\right)=K_{0}(i)\left(\nu\left(n_{2}\right)\right)$ if and only if $m$ divides $n_{1}-n_{2}$. We consider the case of $n_{1} \leqslant 0$ and $n_{2} \geqslant 0$; all other combinations of signs are handled in a similar manner.

By the above results, $K_{0}(i)\left(\nu\left(n_{1}\right)\right)=K_{0}(i)\left(\nu\left(n_{2}\right)\right)$ if and only if $-\left[Q_{-n_{1}}\right]_{0}=\left[Q_{n_{2}}\right]_{0}$. By the definition of $\sim_{0}$, the latter occurs if and only if $Q_{-n_{1}} \oplus Q_{n_{2}} \oplus I^{(k)} \sim_{a} I^{(k)}$ for some $k \in \mathbb{N}$. But the left-hand side is algebraically equivalent to $Q_{s} \oplus I^{(k)}$, where $s=$ $n_{2}-n_{1}$. Equivalently, there exists an operator space $Z$, containing $X^{k}$ as a subspace of codimension $s$ and completely isomorphic to it. Let $n=\lceil s / m\rceil$, and $\ell=m n-s . X^{k}$ is completely isomorphic to all of its subspaces of codimension $m n$, and hence so is $Z$. Therefore, $X^{k}$ is completely isomorphic to its subspace of codimension $\ell$. By Lemma 3.13, $m$ divides $\ell$, and hence $m$ also divides $s$.

\section{Further properties of $X(C, m)$}

\subsection{Subspaces with few completely bounded maps}

We show that the shift 'degenerates' on some subspaces of $X(C, m)$.

Proposition 7.1. For any $n \in \mathbb{N}$ there exists a subspace $Z$ of $X$ such that $Z$ is a direct sum of mutually orthogonal infinite-dimensional subspaces $Z_{1}, \ldots, Z_{n}$, and $T: Z \rightarrow X$ is completely bounded if and only if $T=\sum_{k=1}^{n} c_{k} J_{k}+W$, where $W \in \mathcal{S}_{2}$, and $J_{k}$ is the composition of the orthogonal projection from $Z$ onto $Z_{k}$ and the embedding of $Z_{k}$ into $X$. For such $\left(c_{k}\right)_{k=1}^{n}$ and $W$,

$$
\max \left\{\left|c_{1}\right|, \ldots,\left|c_{n}\right|, \frac{\|W\|_{2}}{21 \sqrt{2} \sqrt{n}}\right\} \leqslant\|T\|_{\mathrm{cb}} \leqslant C^{n}\left(\left|c_{1}\right|+\cdots+\left|c_{n}\right|\right)+\|W\|_{2} .
$$

Moreover, $Z$ is not completely isomorphic to a complete direct sum of more than $n$ infinite-dimensional operator spaces.

The following lemma establishes Proposition 7.1 for $n=1$.

Lemma 7.2. Suppose that $|\lambda|=1$, and a shift lacunary orthonormal sequence $\left(\xi_{i}\right)$ exists such that $\left\|S^{m} \xi_{i}-\lambda \xi_{i}\right\|<\varepsilon_{i}=\alpha^{2 i}(1+i / \alpha)^{-5} 2^{-(2 i+4)}$ for every $i \in \mathbb{N}$. Then $T$ : $Z \rightarrow X$ is completely bounded if and only if $T=c J+W$, where $J$ is the embedding of $Z=\operatorname{span}\left[\xi_{i} \mid i \in \mathbb{N}\right]$ into $X$. Moreover,

$$
\max \left\{|c|, \frac{\|W\|_{2}}{21 \sqrt{2}}\right\} \leqslant\|T\|_{\mathrm{cb}} \leqslant|c|+\|W\|_{2} .
$$

Proof. By a simple induction argument,

$$
\left\|S^{m j} \xi_{i}-\lambda^{j} \xi_{i}\right\| \leqslant\left\|\lambda\left(S^{m(j-1)} \xi_{i}-\lambda^{j-1}\right) \xi_{i}\right\|+\left\|S^{m(j-1)}\left(S^{m} \xi_{i}-\lambda \xi_{i}\right)\right\|<j \varepsilon_{i}
$$

for any $j \in \mathbb{N}$, and therefore, the sequence $\left(\xi_{i}\right)$ is regular, with $\alpha_{j}=\lambda^{j}$.

Consider $T \in \mathrm{CB}(Z, X)$ satisfying $\|T\|_{\mathrm{cb}}<\frac{1}{4}$. Then $T=\left.\tilde{T}_{a}\right|_{Z}+W$, for some $a=$ $\left(a_{j}\right)_{j \in \mathbb{Z}} \in \ell_{1}\left(C^{|j|}, \mathbb{Z}\right)$, such that $\|a\|_{w}<1$ and $\|W\|_{2}<5 \sqrt{2}$. Let $c=\sum_{j} a_{j} \lambda^{j}$. Clearly, 
$|c| \leqslant\|a\|_{w}$. Next we show that

$$
\left\|\left.\tilde{T}_{a}\right|_{Z}-c J\right\|_{2}^{2}=\sum_{i}\left\|\left(\sum_{j} a_{j} S^{m j}-c\right) \xi_{i}\right\|^{2}<\frac{1}{8} .
$$

By (7.1),

$$
\begin{aligned}
\left\|\left(\sum_{j} a_{j} S^{m j}-c\right) \xi_{i}\right\| & =\left\|\sum_{j} a_{j}\left(S^{m j}-\lambda^{j}\right) \xi_{i}\right\| \\
& \leqslant \sum_{|j| \geqslant K_{i}+1}\left|a_{j}\right|\left\|\left(S^{m j}-\lambda^{j}\right) \xi_{i}\right\|+\sum_{|j| \leqslant K_{i}}\left|a_{j}\right|\left\|\left(S^{m j}-\lambda^{j}\right) \xi_{i}\right\| \\
& <2 \sum_{|j| \geqslant K_{i}+1}\left|a_{j}\right|+\|a\|_{w} \sum_{|j| \leqslant K_{i}}|j| \varepsilon_{i} \\
& <C^{-\left(K_{i}+1\right)}+\frac{\alpha^{2 i}}{(1+i / \alpha)^{4} 2^{2 i+4}}<3 \mathrm{e}^{-2 i}
\end{aligned}
$$

(recall that $C^{-\left(K_{i}+1\right)} \leqslant C^{-2 i \ln C}=\mathrm{e}^{-2 i}$ ). Therefore,

$$
\left\|\left.\tilde{T}_{a}\right|_{Z}-c J\right\|_{2}^{2}=\sum_{i=1}^{\infty}\left\|\left(\tilde{T}_{a}-c\right) \xi_{i}\right\|^{2}<9 \sum_{i=1}^{\infty} \mathrm{e}^{-4 i},
$$

which establishes (7.2). Thus,

$$
\|T-c J\|_{2} \leqslant\left\|T-\tilde{T}_{a}\right\|_{2}+\left\|\tilde{T}_{a}-c J\right\|_{2} \leqslant 5 \sqrt{2}+\frac{1}{2 \sqrt{2}} .
$$

To finish the proof, observe that $|c| \leqslant\|T\|$.

Proof of Proposition 7.1. For $1 \leqslant k \leqslant n$ let $\lambda_{k}=\mathrm{e}^{2 \pi \sqrt{-1}(k-1) / n}$. Select a shift lacunary sequence $\left(\xi_{i}\right)$ such that $\left\|\lambda_{k} \xi_{i}-S^{m} \xi_{i}\right\|<\alpha^{2 i}(1+i / \alpha)^{-5} 2^{-(2 i+4)}$ whenever $i=$ $n(\ell-1)+k$, for some $\ell \in \mathbb{N}$. Let $Z_{k}=\operatorname{span}\left[\xi_{n(\ell-1)+k} \mid \ell \in \mathbb{N}\right]$, and $Z=\operatorname{span}\left[\xi_{i} \mid i \in\right.$ $\mathbb{N}]=\operatorname{span}\left[Z_{1}, \ldots, Z_{n}\right]$.

Suppose $T \in \mathrm{CB}(Z, X)$. By Lemma 7.2, for every $k$ there exist $c_{k} \in \mathbb{C}$ and $W_{k} \in \mathcal{S}_{2}$ such that $\left|c_{k}\right| \leqslant\left\|\left.T\right|_{Z_{k}}\right\| \leqslant\|T\|,\left\|W_{k}\right\|_{2} \leqslant 476\|T\|_{\text {cb }}$ and $\left.T\right|_{Z_{k}}=c_{k} J_{k}+W_{k}$. The spaces $\left(Z_{k}\right)$ are mutually orthogonal, and hence

$$
\left\|T-\sum_{k=1}^{n} c_{k} J_{k}\right\|_{2}=\left(\sum_{k=1}^{n}\left\|W_{k}\right\|_{2}^{2}\right)^{1 / 2} \leqslant 476 \sqrt{n}\|T\|_{\mathrm{cb}} .
$$

On the other hand, by the proof of Lemma 7.2,

$$
\left\|\left.S^{m j}\right|_{Z}-\sum_{k=1}^{n} \lambda_{k}^{j} J_{k}\right\|_{2}=\sum_{k=1}^{n} \sum_{\ell=0}^{\infty}\left\|\left(S^{m j}-\lambda_{k}^{j}\right) \xi_{n \ell+k}\right\|^{2}<9 \sum_{i=1}^{\infty} \mathrm{e}^{-4 i}<1
$$


for $0 \leqslant j \leqslant n-1$. Therefore, $\left\|\sum_{k=1}^{n} \lambda_{k}^{j} J_{k}\right\|_{\mathrm{cb}} \leqslant 1+\left\|S^{m j}\right\|_{\mathrm{cb}}<C^{n}$. However,

$$
J_{i}=\frac{1}{n} \sum_{j=0}^{n-1} \bar{\lambda}_{i}^{j} \sum_{k=1}^{n} \lambda_{k}^{j} J_{k},
$$

and hence $\left\|J_{i}\right\|_{\mathrm{cb}} \leqslant C^{n}$ for $1 \leqslant i \leqslant n$.

Now suppose that $R_{1}, \ldots, R_{n+1}$ are completely bounded projections in $Z$ and that $R_{i} R_{j}=0$ if $i \neq j$. We must show that at least one of the projections has finite-dimensional range. To this end, define a map $\pi: \mathrm{CB}(Z) \rightarrow \ell_{\infty}^{n}$ by setting

$$
\pi\left(\sum_{k=1}^{n} c_{k} J_{k}+W\right)=\left(c_{k}\right)_{k=1}^{n}
$$

(here $\left(c_{k}\right) \subset \mathbb{C}$, and $\left.W \in \mathcal{S}_{2}\right)$. By the above, $\pi$ is well defined and is a bounded algebraic homomorphism. Therefore, $\pi\left(R_{i}\right)=\chi_{E_{i}}$ for any $i$ (here $E_{i}$ is a subset of $\{1, \ldots, n\}$ ). Moreover,

$$
\chi_{E_{i}} \chi_{E_{j}}=\pi\left(R_{i}\right) \pi\left(R_{j}\right)=\pi\left(R_{i} R_{j}\right)=\pi(0)=0,
$$

and therefore, by the pigeon-hole principle, $E_{i}$ is empty for some $i$. For such $i, R_{i}$ is Hilbert-Schmidt, and hence of finite rank.

\subsection{Compact operators on subspaces of $X(C, m)$}

We conclude the paper by pointing out that, for a generic subspace $Y \hookrightarrow X=X(C, m)$, compactness of an operator $T \in \mathrm{CB}(Y, X)$ does not imply any further 'interesting' properties of $T$ (cf. Lemma 4.3). First show that subspaces of $X$ allow a large variety of diagonal operators.

Proposition 7.3. Suppose the function $f: \mathbb{A} \rightarrow \mathbb{C}$ belongs to $\mathcal{Y}$, and $\left\{\lambda_{1}, \lambda_{2}, \ldots\right\} \subset$ $f(\mathbb{T})$. There then exists a subspace $Y$ of $X$, spanned by a shift lacunary basis $\left(\xi_{i}\right)_{i \in \mathbb{N}}$, and $T \in \mathrm{CB}(X)$, so that $T \xi_{i}=\lambda_{i} \xi_{i}$ for any $i \in \mathbb{N}$.

Proof. Find the points $z_{1}, z_{2}, \cdots \in \mathbb{T}$ such that $f\left(z_{n}\right)=\lambda_{n}$ for any $n \in \mathbb{N}$. By approximating 'very spiky' functions concentrated around the points $z_{n}$ by trigonometric polynomials, we find that, for each $n$,

$$
g_{n}(z)=\sum_{j=-K_{n}}^{K_{n}} \alpha_{j n} z^{j}
$$

such that $\left\|g_{n}\right\|_{2}=1$, and $\left\|\left(f-\lambda_{n}\right) g_{n}\right\|_{2}<2^{-n}$. Find an increasing sequence $\left(M_{n}\right)$ of positive integers such that $M_{n}>K_{n}$ for every $n$, and moreover, the sequence $\left(\xi_{n}\right)$ is shift lacunary, where

$$
\xi_{n}=\sum_{j=-K_{n}}^{K_{n}} \alpha_{j n} \delta_{m\left(M_{n}+j\right)} .
$$


Write $f(z)=\sum_{j \in \mathbb{Z}} a_{j} z^{j}$. By the above, $\left\|\tilde{T}_{a} \xi_{n}-\lambda_{n} \xi_{n}\right\|<2^{-n}$. Define an operator $W$ : $X \rightarrow X$ by setting

$$
W \xi_{n}=\tilde{T}_{a} \xi_{n}-\lambda_{n} \xi_{n} \quad \text { and }\left.\quad W\right|_{\operatorname{span}\left[\xi_{n} \mid n \in \mathbb{N}\right]^{\perp}}=0 .
$$

Clearly, $W$ is Hilbert-Schmidt, and hence $\tilde{T}_{a}-W \in \mathrm{CB}(X)$. However, $\left(\tilde{T}_{a}-W\right) \xi_{n}=$ $\lambda_{n} \xi_{n}$.

It easily follows from the above proposition that, for any separable symmetric sequence space $\mathcal{E}$ not isomorphic to $c_{0}$, there exists a subspace $Y$ of $X$ and a compact $T \in \mathrm{CB}(X)$ such that the sequence of singular numbers $\left(\sigma_{i}(T)\right)_{i \in \mathbb{N}}$ does not belong to $\mathcal{E}$ (in other words, such a $T$ does not belong to the ideal $\mathcal{S}_{\mathcal{E}}$; see, for example, [7] for the definition). Indeed, we find a sequence $\left(s_{n}\right)_{n \in \mathbb{N}} \in c_{0} \backslash \mathcal{E}$ such that $s_{n} \in\left(0, \frac{1}{2}\right)$ for any $n$, and consider $f(z)=1-z$. We then find a sequence $\left(z_{n}\right)$ such that $\left|f\left(z_{n}\right)\right|=s_{n}$. By Proposition 7.3, there exists $T \in \mathrm{CB}\left(\operatorname{span}\left[\xi_{i} \mid i \in \mathbb{N}\right]\right)$ such that $T \xi_{i}=f\left(z_{i}\right) \xi_{i}$. Then $\left(s_{n}\right)$ is the sequence of singular numbers of the operator $T$.

Several versions of 'non-commutative compactness' have been put forward over the years (see, for example, $[\mathbf{1 7}, \mathbf{1 8}])$. If $u \in \mathrm{CB}(Y, Z)$ ( $Y$ and $Z$ are operator spaces), one can introduce the sequence of complete Kolmogorov numbers by setting, for $n \in \mathbb{N}$,

$$
d_{n}^{\mathrm{cb}}(u)=\inf \left\{\left\|q_{F} u\right\|_{\mathrm{cb}} \mid F \hookrightarrow Z, \operatorname{dim} F \leqslant n\right\}
$$

(here $q_{F}: Z \rightarrow Z / F$ is a quotient map). Similarly, the complete Gelfand numbers are defined by setting

$$
c_{n}^{\mathrm{cb}}(u)=\inf \left\{\left\|u j_{G}\right\|_{\mathrm{cb}} \mid G \hookrightarrow Y, \operatorname{dim} Y / G \leqslant n\right\},
$$

where $j_{G}$ is the canonical embedding. A map $u \in \mathrm{CB}(Y, Z)$ is called operator compact (Gelfand compact) if $\lim _{n} d_{n}^{\mathrm{cb}}(u)=0$ (respectively, $\lim _{n} c_{n}^{\mathrm{cb}}(u)=0$ ).

Below we show that a compact $T \in \mathrm{CB}(Y, X)$ need not be either operator or Gelfand compact.

Proposition 7.4. There exist a subspace $Y$ of the space $X=X(C, m)$ and a compact operator $T \in \mathrm{CB}(Y)$ such that

$$
\inf \left\{\left\|q_{F} T j_{G}\right\|_{\mathrm{cb}} \mid F \hookrightarrow X, G \hookrightarrow Y, \operatorname{dim} F<\infty, \operatorname{dim} Y / G<\infty\right\}>0 .
$$

Remark 7.5. A fortiori, $T$ cannot be approximated by finite-rank operators in the c.b. norm. This contrasts sharply with operators on $X$ : among the compact operators on $X$ itself, the norms $\|\cdot\|_{\mathrm{cb}}$ and $\|\cdot\|_{2}$ are equivalent. Hence, for any compact $T \in \mathrm{CB}(X)$ there exists a sequence of finite-rank maps $\left(T_{n}\right)$ such that

$$
\lim _{n}\left\|T-T_{n}\right\|_{\mathrm{cb}}=\lim _{n}\left\|T-T_{n}\right\|_{2}=0 .
$$

The following technical lemma seems to be part of the folklore. We state and prove it for the sake of completeness. 
Lemma 7.6. Suppose that $Y$ and $Z$ are operator spaces, $u \in \mathrm{CB}(Y, Z)$, and there exists a net $\left(F_{i}\right)$ of finite-dimensional subspaces of $Z$, such that $Z=\overline{\bigcup_{i} F_{i}}$. Then

$$
\inf \left\{\left\|q_{F} u\right\|_{\mathrm{cb}} \mid F \hookrightarrow X, \operatorname{dim} F<\infty\right\}=\inf _{i}\left\|q_{F_{i}} u\right\|_{\mathrm{cb}} .
$$

Proof. Assume, without loss of generality, that $\|u\|_{\mathrm{cb}} \leqslant \frac{1}{3}$. Let

$$
\lambda=\inf \left\{\left\|q_{F} u\right\|_{\mathrm{cb}} \mid F \hookrightarrow X, \operatorname{dim} F<\infty\right\} .
$$

For any $\varepsilon \in\left(0, \frac{1}{3}\right)$, there exists a finite-dimensional subspace $F \hookrightarrow Z$ such that $\left\|q_{F} u\right\|_{\mathrm{cb}}<$ $\lambda+\varepsilon$. Then for every $x \in X \otimes \mathcal{K}$ with $\|x\|<1$ there exists $f \in F \otimes \mathcal{K}$ satisfying

$$
\left\|f+\left(u \otimes I_{\mathcal{K}}\right) x\right\|<\lambda+\varepsilon .
$$

By the triangle inequality, $\|f\|<1$. A simple perturbation argument (see $[\mathbf{2 3}, \S 2.13]$ ) shows that there exists $i$ such that for any $f \in F \otimes \mathcal{K}$ there exists $f^{\prime} \in F_{i} \otimes \mathcal{K}$, satisfying $\left\|f-f^{\prime}\right\|<\varepsilon$. By the triangle inequality, $\left\|f^{\prime}+\left(u \otimes I_{\mathcal{K}}\right) x\right\|<\lambda+2 \varepsilon$. Hence, $\left\|q_{F_{i}} u\right\|_{\mathrm{cb}} \leqslant \lambda+2 \varepsilon$. However, $\varepsilon$ can be taken to be arbitrarily small.

Proof of Propisition 7.4. Find a sequence $\left(\ell_{i}\right)$ of positive integers such that $m \leqslant$ $\ell_{1} \leqslant \ell_{2} \leqslant \cdots, \lim _{i} \ell_{i}=\infty$ and $\sum_{i} 1 / \ell_{i}=\infty$. Consider a shift lacunary sequence $\left(\xi_{i}\right)_{i \in \mathbb{N}}$, consisting of vectors

$$
\xi_{i}=\frac{1}{\sqrt{\ell_{i}}} \sum_{k=N_{i}+1}^{N_{i}+\ell_{i}} \delta_{k},
$$

where $N_{k}+\ell_{k}+2 m(1+\lfloor k / \alpha\rfloor)<N_{k+1}$ for any $k$ (as before, $\alpha=\min \{1, \ln C\} / 2$ ). We shall show that $Y=\operatorname{span}\left[\xi_{i} \mid i \in \mathbb{N}\right] \hookrightarrow X$ and $T=\left.\left(I-S^{m}\right)\right|_{Y}$ satisfy the conditions of Theorem 7.4. Clearly, $\|T\|_{\mathrm{cb}} \leqslant 1+C$. Moreover, $T \xi_{i}=\sqrt{2 m / \ell_{i}} \eta_{i}$, where

$$
\eta_{i}=\frac{1}{\sqrt{2 m}} \sum_{j=0}^{m-1}\left(\delta_{N_{i}+j}-\delta_{N_{i}+\ell_{i}+j}\right) .
$$

Note that $\lim _{i}\left\|T \xi_{i}\right\|=0$ and the vectors $\eta_{i}$ are orthonormal; hence, $T$ is compact.

Applying Lemma 7.6 to $T$ and then to $T^{*}$, we see that it suffices to show that, for any $K \in \mathbb{N}$,

$$
\left\|\left.q_{K} T\right|_{Y_{K}}\right\|_{\mathrm{cb}} \geqslant \frac{1}{2}
$$

where $Y_{K}=\operatorname{span}\left[\xi_{i} \mid i>K\right], F_{K}=\operatorname{span}\left[\delta_{1}, \ldots, \delta_{K}\right]$ and $q_{K}: X \rightarrow X / F_{K}$ is the quotient map. To this end, fix $M \in \mathbb{N}$ so that

$$
c=\left(\sum_{i=K+1}^{K+M} 1 / \ell_{i}\right)^{-1 / 2}<(2 m)^{-1} .
$$

Define $v \in B\left(\ell_{2}^{M}, Y_{K}\right)$ and $u \in B\left(X, \ell_{2}^{M}\right)$ by setting $v e_{i}=\xi_{K+i}$, and $u^{*} e_{i}=c_{i} \eta_{K+i}$, where $\left(e_{i}\right)_{i=1}^{M}$ is the canonical basis for $\ell_{2}^{M}, c_{i}=c / \sqrt{\ell_{K+i}}$. Note that $\|v\|=1=\|u\|_{2}$. Pick $\varepsilon \in(0,1)$ and find (as in the proof of Lemma 2.2) $s>\varepsilon^{-1}$ such that $n_{s}=M$, 
and $\left\|u_{s}-u\right\|_{1}<\varepsilon$. We identify the domain of $v$ and the range of $u$ with $E_{s}^{*}$ and $E_{s}$, respectively. Note that $F_{K}=\operatorname{ker} q_{K} \subset \operatorname{ker} u$, and hence there exists a linear operator $\tilde{u} \in B\left(X / F_{K}, E_{s}\right)$ such that $\tilde{u} q_{K}=u$. We obviously have

$$
\left\|\left.q_{K} T\right|_{Y_{K}}\right\|_{\mathrm{cb}} \geqslant \frac{\left\|\tilde{u} q_{K} T v\right\|_{\mathrm{cb}}}{\|\tilde{u}\|_{\mathrm{cb}}\|v\|_{\mathrm{cb}}}
$$

To establish (7.3), we find suitable estimates for the numerator and the denominator of (7.4). Look at the numerator first. Recall that $\tilde{u} q_{K}=u$. By the properties of the space $E_{s}($ see $\S 2)$,

$$
\begin{aligned}
(4+\varepsilon)\left\|\tilde{u} q_{K} T v\right\|_{\mathrm{cb}} \geqslant\|u T v\|_{1} & =\sum_{i=1}^{M}\left|\left\langle T v e_{i}, u^{*} e_{i}\right\rangle\right|=\sum_{i=1}^{M}\left|\left\langle\sqrt{\frac{2 m}{\ell_{K+i}}} \eta_{K+i}, c_{i} \eta_{K+i}\right\rangle\right| \\
& =\sum_{i=1}^{M} \frac{\sqrt{2 m}}{\ell_{K+i}} c=\sum_{i=1}^{M} \frac{\sqrt{2 m}}{\ell_{K+i}}\left(\sum_{i=1}^{M} \frac{1}{\ell_{K+i}}\right)^{-1 / 2} \\
& =\frac{\sqrt{2 m}}{c}
\end{aligned}
$$

Next we show that $\|v\|_{\mathrm{CB}\left(E_{s}^{*}, X\right)}<\sqrt{m} /(\sqrt{2} c)+\varepsilon$. As in the proof of Lemma 2.2,

$$
\|v\|_{\mathrm{cb}} \leqslant \max \left\{1, \sup _{p, q \geqslant 0} C^{-(p+q)}\left\|u_{s} S^{m p} S^{* m q} v\right\|_{1}\right\} .
$$

However,

$$
\begin{aligned}
\left\|u S^{m p} S^{* m q} v\right\|_{1} & \leqslant \sum_{i, j=1}^{M}\left|\left\langle u^{*} e_{i}, S^{m p} S^{* m q} v e_{j}\right\rangle\right| \\
& =\sum_{i=1}^{M}\left|\left\langle u^{*} e_{i}, S^{m p} S^{* m q} v e_{i}\right\rangle\right|+\sum_{i \neq j}\left|\left\langle u^{*} e_{i}, S^{m p} S^{* m q} v e_{j}\right\rangle\right| .
\end{aligned}
$$

The first term on the right can be estimated as follows:

$$
\sum_{i=1}^{M}\left|\left\langle u^{*} e_{i}, S^{m p} S^{* m q} v e_{i}\right\rangle\right|=\sum_{i=1}^{M}\left|\left\langle c_{i} \eta_{i+K}, S^{m p} S^{* m q} \xi_{i+K}\right\rangle\right| \leqslant \sum_{i=1}^{M} \frac{c_{i} m}{\sqrt{2 m} \sqrt{\ell_{K+i}}}=\frac{\sqrt{m}}{\sqrt{2} c} .
$$

As for the second term, the conditions on the sequence $\left(N_{k}\right)$ imply that

$$
\left\langle u^{*} e_{i}, S^{m p} S^{* m q} v e_{j}\right\rangle=\left\langle c_{i} \eta_{i+K}, S^{m p} S^{* m q} \xi_{j+K}\right\rangle=0
$$

whenever $i \neq j$, and $\max \{i, j\} \leqslant \alpha(p+q)$. Therefore,

$$
\sum_{i \neq j}\left|\left\langle u e_{i}, S^{m p} S^{* m q} v e_{j}\right\rangle\right| \leqslant\left|\left\{(i, j) \in \mathbb{N}^{2} \mid i \neq j, \max \{i, j\} \leqslant \alpha(p+q)\right\}\right| \leqslant(\alpha(p+q))^{2} .
$$


Thus,

$$
\begin{aligned}
\left\|u_{s} S^{m p} S^{* m q} v\right\|_{1} & \leqslant\left\|u_{s}-u\right\|_{1}+\left\|u S^{m p} S^{* m q} v\right\|_{1} \\
& <\varepsilon+\frac{\sqrt{m}}{\sqrt{2} c}+(\alpha(p+q))^{2} \\
& <\varepsilon+\frac{\sqrt{m}}{\sqrt{2} c}(1+\alpha(p+q))^{2} \\
& \leqslant \varepsilon+\frac{\sqrt{m}}{\sqrt{2} c} C^{p+q}
\end{aligned}
$$

and therefore $\|v\|_{\mathrm{cb}} \leqslant \varepsilon+\sqrt{m} / \sqrt{2} c$.

Finally, by definition of the quotient of operator spaces,

$$
\|\tilde{u}\|_{\mathrm{cb}}=\|u\|_{\mathrm{cb}} \leqslant\left\|u_{s}\right\|_{\mathrm{cb}}+\left\|u-u_{s}\right\|_{\mathrm{cb}}<1+\varepsilon
$$

(here we use (2.1)). Thus, (7.4) yields

$$
\|T\|_{\mathrm{cb}} \geqslant \frac{\sqrt{2 m} / c}{(4+\varepsilon)(\varepsilon+\sqrt{m} / \sqrt{2} c)(1+\varepsilon)} .
$$

However, $\varepsilon>0$ can be arbitrarily small. Hence, we have (7.3).

\section{Representations of $\ell_{1}\left(C^{|j|}, \mathbb{Z}\right)$ and $\ell_{1}\left(C^{j}, \mathbb{Z}^{+}\right)$}

In this section, we modify the construction presented above to exhibit operator spaces arising from faithful unital representations of weighted convolution algebras $\ell_{1}\left(C^{|j|}, \mathbb{Z}\right)$ and $\ell_{1}\left(C^{j}, \mathbb{Z}^{+}\right)$. As before, $C>1$ and $m \in \mathbb{N}$ are fixed. We shall use $S_{\mathrm{b}}$ to denote the bilateral shift on $\ell_{2}(\mathbb{Z})$. For the semigroup $\Gamma=\mathbb{Z}$ or $\mathbb{Z}^{+}=\mathbb{N} \cup\{0\}$, consider the following contractive unital representations:

$$
\begin{aligned}
\pi_{\mathbb{Z}, \mathrm{b}}: \ell_{1}\left(C^{|j|}, \mathbb{Z}\right) \rightarrow B\left(\ell_{2}(\mathbb{Z})\right): \delta_{j} \mapsto C^{-|j|} S_{\mathrm{b}}^{m j} ; \\
\pi_{\mathbb{Z}^{+}, \mathrm{b}}: \ell_{1}\left(C^{j}, \mathbb{Z}^{+}\right) \rightarrow B\left(\ell_{2}(\mathbb{Z})\right): \delta_{j} \mapsto C^{-|j|} S_{\mathrm{b}}^{m j} ; \\
\pi_{\mathbb{Z}^{+}, \mathrm{r}}: \ell_{1}\left(C^{j}, \mathbb{Z}^{+}\right) \rightarrow B\left(\ell_{2}\right): \delta_{j} \mapsto C^{-|j|} S^{m j} ; \\
\pi_{\mathbb{Z}^{+}, \ell}: \ell_{1}\left(C^{j}, \mathbb{Z}^{+}\right) \rightarrow B\left(\ell_{2}\right): \delta_{j} \mapsto C^{-|j|} S^{* m j} .
\end{aligned}
$$

Here, the letters $\mathrm{b}, \ell$ and $\mathrm{r}$ stand for 'bilateral', 'left' and 'right', respectively. It turns out that these representations give rise to operator spaces.

Theorem 8.1. Suppose that $C>1, m \in \mathbb{N},(\Gamma, d) \in\left\{(\mathbb{Z}, b),\left(\mathbb{Z}^{+}, b\right),\left(\mathbb{Z}^{+}, r\right),\left(\mathbb{Z}^{+}, \ell\right)\right\}$, and $\pi_{\Gamma, d}$ is one of the representations described above. Let $\Gamma^{\prime}=\mathbb{Z}$ if $\Gamma=\mathbb{Z}$ and let $\Gamma^{\prime}=\mathbb{N}$ if $\Gamma=\mathbb{Z}^{+}$. There then exists an operator space $X_{\Gamma, d}=X_{\Gamma, d}(C, m)$, isometric to $\ell_{2}\left(\Gamma^{\prime}\right)$, such that $\pi_{\Gamma, d}$ induces an isomorphism from $\ell_{1}\left(C^{|j|}, \Gamma\right)$ into $\mathrm{CB}\left(X_{\Gamma, d}\right)$. Moreover, the following conditions hold. 
(i) For any $a \in \ell_{1}\left(C^{|j|}, \Gamma\right),\left\|\pi_{\Gamma, d}(a)\right\|_{\mathrm{cb}} \geqslant \frac{1}{4}\|a\|_{w}$.

(ii) $T \in \mathrm{CB}\left(X_{\Gamma, d}\right)$ if and only if $T=\pi_{\Gamma, d}(a)+W$ for some $a \in \ell_{1}\left(C^{|j|}, \Gamma\right)$ and $W \in \mathcal{S}_{2}$. In this case,

$$
\|a\|_{w}+\|W\|_{2} \geqslant\|T\|_{\mathrm{cb}} \geqslant \max \left\{\|a\|_{w} / 4, \gamma\|W\|_{2}\right\},
$$

where $\gamma$ depends only on $C$ and $m$.

(iii) If $Y$ is an infinite-dimensional subspace of $X_{\Gamma, d}$, then for any $T \in \operatorname{CB}\left(Y, X_{\Gamma, d}\right)$ there exists $a \in \ell_{1}\left(C^{|j|}, \Gamma\right)$ such that $T-\left.\pi_{\Gamma, d}(a)\right|_{Z}$ is compact, and $\|a\|_{w} \leqslant$ $4\|T\|_{\mathrm{cb}}$. Moreover, $Y$ contains an infinite-dimensional subspace $Z$ such that any $T \in \mathrm{CB}\left(Z, X_{\Gamma, d}\right)$ can be written as $T=\left.\pi_{\Gamma, d}(a)\right|_{Z}+W$, with $a \in \ell_{1}\left(C^{|j|}, \Gamma\right)$, $W \in \mathcal{S}_{2},\|W\|_{2} \leqslant 20 \sqrt{2}\|T\|_{\mathrm{cb}}$ and $\|a\|_{w} \leqslant 4\|T\|_{\mathrm{cb}}$.

(iv) Any infinite-dimensional subspace of $X_{\Gamma, d}$ fails the OAP.

(v) $X_{\Gamma, d}$ is not completely isomorphic to any of its proper subspaces.

(vi) $K_{0}\left(\mathrm{CB}\left(X_{\Gamma, d}\right)\right)=\mathbb{Z}^{2}, K_{1}\left(\mathrm{CB}\left(X_{\mathbb{Z}^{+}, d}\right)\right)=\{0\}$ and $K_{1}\left(\mathrm{CB}\left(X_{\mathbb{Z}, \mathrm{b}}\right)\right)=\mathbb{Z}$.

The proof of this theorem is similar to those presented above, so we outline it only briefly. We use the spaces $E_{i}$ described in $\S 2$. The maps $u_{i}: \ell_{2}\left(\Gamma^{\prime}\right) \rightarrow E_{i}$ are such that, for all $n, N \in \mathbb{N}$ and $u: \ell_{2}\left(\Gamma^{\prime}\right) \rightarrow \ell_{2}^{n}$ with $\|u\|_{2}=1$, there exists $i>N$ such that $\operatorname{dim} E_{i}=n$ and $\left\|u-u_{i}\right\|_{1}<2^{-N}$. We define the operator space structure, for $x \in \ell_{2}\left(\Gamma^{\prime}\right) \otimes \mathcal{K}$, by setting

$$
\|x\|_{X_{\Gamma, d} \otimes \mathcal{K}}=\sup \left\{C^{-|j|}\left\|\left(u_{i} S_{d}^{m j} \otimes I_{\mathcal{K}}\right) x\right\|_{E_{i} \otimes \mathcal{K}} \mid i \in \mathbb{N}, j \in \Gamma\right\},
$$

where $S_{d}$ stands for $S_{\mathrm{b}}(d=\mathrm{b}), S(d=\mathrm{r})$, or $S^{*}(d=\ell)$. Then $X_{\Gamma, d}$ is an operator space, isometric to $\ell_{2}\left(\Gamma^{\prime}\right)$, and $\left\|\pi_{\Gamma, d}(a)+W\right\|_{\mathrm{cb}} \leqslant\|a\|_{w}+\|W\|_{2}$ for any $a \in \ell_{1}\left(C^{|j|}, \Gamma\right)$ and $W \in \mathcal{S}_{2}$.

For notational simplicity, we henceforth use $X$ and $\pi$ for $X_{\Gamma, d}$ and $\pi_{\Gamma, d}$, respectively.

Our next goal is to split an operator $T \in \mathrm{CB}(Y, X), Y \hookrightarrow X$, into its compact and Toeplitz parts, thus proving (iii). When dealing with right or left shifts $(d=\mathrm{r}$ or $\ell$ ), we use shift lacunary sequences as before. In the bilateral case we employ bilateral shift lacunary sequences. We say that a sequence $\left(\xi_{i}\right)_{i \in \mathbb{N}}$ in $\ell_{2}(\mathbb{Z})$ is bilaterally shift lacunary if it is normalized, and there exists a sequences of mutually orthogonal subspaces $\left(F_{i}\right)_{i \in \mathbb{N}}$ of $\ell_{2}(\mathbb{Z})$, such that $\operatorname{dim} F_{i}=2 K_{i}+1, S_{\mathrm{b}}^{m j} \xi_{i} \in F_{i}$ whenever $|j| \leqslant K_{i}$, if $i \neq k$. As in Proposition 3.4, we prove: suppose $\left(Y_{n}\right)$ is a sequence of infinite-dimensional subspaces of $\ell^{2}(\mathbb{Z})$. There then exists a bilaterally shift lacunary sequence $\left(\xi_{n}\right)_{n \in \mathbb{N}}$, such that $\xi_{n} \in Y_{n}$ for any $n$. As in $\S 3$, we show that, for any $Y \hookrightarrow X$ and $T \in \mathrm{CB}(Y, X)$, there exists $a \in \ell_{1}\left(C^{|j|}, \Gamma\right)$ for which $T-\left.\pi(a)\right|_{Y}$ is compact.

As in $\S \S 4$ and 5 , we define $c$-nice and regular sequences, and establish parts (i), (ii) and (iv) of Theorem 8.1.

To deal with parts (v) and (vi), it is convenient to represent $\ell_{1}\left(C^{|j|}, \Gamma\right)$ as a function algebra $\mathcal{Y}_{\Gamma}$. More precisely, by [4, Theorems 4.6.9 and 4.6.12], the spectrum of $\ell_{1}\left(C^{|j|}, \Gamma\right)$ is

$$
\mathbb{A}_{\Gamma}= \begin{cases}\left\{z \in \mathbb{C}\left|C^{-1} \leqslant\right| z \mid \leqslant C\right\}, & \Gamma=\mathbb{Z}, \\ \{z \in \mathbb{C}|| z \mid \leqslant C\}, & \Gamma=\mathbb{Z}^{+} .\end{cases}
$$


$\mathcal{Y}_{\Gamma}$ is the algebra of functions $f$ on $\mathbb{A}_{\Gamma}$ with the power series decomposition

$$
f(z)=\sum_{j \in \Gamma} a_{j} z^{j}
$$

and the norm

$$
\|f\|=\sum_{j \in \Gamma}\left|a_{j}\right| C^{|j|}
$$

As in $\S 3$, define $\phi: \mathrm{CB}(X) \rightarrow \mathcal{Y}_{\Gamma}$ by setting $\phi(T)(z)=\sum_{j \in \Gamma} a_{j} z^{j}$, where $a=\left(a_{j}\right)_{j \in \Gamma}$ is such that $T-\pi(a)$ is compact (there are no non-trivial compact Toeplitz operators, and hence such an $a$ is unique).

We can now show that $X^{n}=X \oplus \cdots \oplus X$ ( $n$ times) is not completely isomorphic to any of its proper subspaces (this is a counterpart of Lemma 3.13, and yields part (v) of our theorem). To minimize the clutter, we use $\phi_{n}$ and $\pi_{n}$ for $I_{M_{n}} \otimes \phi$ and $I_{M_{n}} \otimes \pi$, respectively. Suppose that $T \in \mathrm{CB}\left(X^{n}\right)$ is a complete isomorphism onto its range. As in the proof of Lemma 3.11, $T$ is Fredholm. Thus, the range of $T$ has finite codimension, and there exists $T_{1} \in \mathrm{CB}\left(X^{n}\right)$ such that $T_{1} T=I_{X^{n}}$. Then $f_{1}(z) f(z)=I_{M_{n}}$ for any $z \in \mathbb{A}_{\Gamma}$, where $f_{1}=\phi_{n}\left(T_{1}\right)$ and $f=\phi_{n}(T) . \pi_{n}$ is a unital representation. Hence, $\pi_{n}(f)$ is invertible. But $T-\pi_{n}(f)$ is compact, and hence $T$ has Fredholm index 0 .

Part (vi) of our theorem can be deduced from (v) by imitating $\S 6$. Alternatively, one can observe that $\phi \circ \pi=I_{\mathcal{Y}_{\Gamma}}$. Hence, the short exact sequence

$$
0 \rightarrow \mathcal{S}_{2} \stackrel{i}{\rightarrow} \mathrm{CB}(X) \stackrel{\phi}{\rightarrow} \mathcal{Y}_{\Gamma} \rightarrow 0
$$

splits, and therefore, $K_{s}(\mathrm{CB}(X))=K_{s}\left(\mathcal{S}_{2}\right) \oplus K_{s}\left(\mathcal{Y}_{\Gamma}\right)$ for $s=0,1[\mathbf{3}$, Proposition 8.3.6]. The $K$-groups of $\mathcal{S}_{2}$ have already been described in Lemma 6.4, while $K_{s}\left(\mathcal{Y}_{\Gamma}\right)=$ $K_{s}\left(C\left(\mathbb{A}_{\Gamma}\right)\right)$, by [27]. But $K_{0}\left(C\left(\mathbb{A}_{\Gamma}\right)\right)=\mathbb{Z}$ (this follows from [3, Example 5.3.2] or [10, Proposition 8.3.1]). As noted in $\S 6, K_{1}\left(C\left(\mathbb{A}_{\mathbb{Z}}\right)\right)=\mathbb{Z}$, while it is easy to show that $K_{1}\left(C\left(\mathbb{A}_{\mathbb{Z}^{+}}\right)\right)=\{0\}$ (see [13, Example 9.6.1]).

Acknowledgements. The author was partly supported by NSF Grant no. DMS0200714. He thanks the organizers of the workshop on Linear Analysis and Probability in College Station, TX, where part of this work was carried out. He is grateful to R. Douglas, B. Maurey and A. Zsák for fruitful discussions on $K$-theory. Last but not least, he thanks the referee for useful advice on better organization of the paper and expanding its scope.

\section{References}

1. E. Berkson and H. Porta, Representations of B(X), J. Funct. Analysis 3 (1969), 1-34.

2. R. Bhatia, Matrix analysis (Springer, 1997).

3. B. BlackadaR, $K$-theory for operator algebras, 2nd edn (Cambridge University Press, 1998).

4. H. DALES, Banach algebras and automatic continuity (Oxford University Press, 2000).

5. R. Douglas, Banach algebra techniques in the theory of Toeplitz operators (American Mathematical Society, Providence, RI, 1972).

6. E. Effros and Z.-J. Ruan, Operator spaces (Oxford University Press, 2000). 
7. I. GOHBERG AND M. KREIN, Introduction to the theory of linear nonselfadjoint operators (American Mathematical Society, Providence, RI, 1969).

8. T. Gowers And B. Maurey, Banach spaces with small spaces of operators, Math. Ann. 307 (1997), 543-568.

9. G. JAMESON, Summing and nuclear norms in Banach space theory (Cambridge University Press, 1987).

10. F. Larsen, N. Laustsen and M. Rørdam, An introduction to $K$-theory for $C^{*}$-algebras (Cambridge University Press, 2000).

11. N. Laustsen, $K$-theory for algebras of operators on Banach spaces. J. Lond. Math. Soc. 59 (1999), 715-728.

12. J. Lindenstrauss And L. Tzafriri, Classical Banach spaces, I (Springer, 1977).

13. B. Maurey, Notes from a series of lectures (held at Spetses, Greece, summer 1994) on HI spaces (available at http://math.jussieu.fr/ maurey/articles/index.html).

14. B. MAUREY, Banach spaces with few operators, in Handbook of the geometry of Banach spaces, Volume 2, pp. 1247-1297 (North-Holland, Amsterdam, 2003).

15. G. Murphy, Topological and analytical indices in $C^{*}$-algebras, J. Funct. Analysis 234 (2006), 261-276.

16. M. Neufang, Z.-J. RuAn And N. Spronk, Completely isometric representations of $M_{\mathrm{cb}} A(G)$ and $U \mathrm{CB}(\hat{G})^{*}$, Trans. Am. Math. Soc., in press.

17. T. OikHBerg, Geometry of operator spaces and products of orthogonal projections, $\mathrm{PhD}$ dissertation, Texas A\&M University (1998).

18. T. OikhBerg, Direct sums of operator spaces, J. Lond. Math. Soc. 64 (2001), 144-160.

19. T. OikhBerg, Operator spaces with prescribed sets of completely bounded maps, $J$. Funct. Analysis 224 (2005), 296-315.

20. T. OIKHBERG, Operator spaces with complete bases, lacking completely unconditional bases, Houston J. Math., in press.

21. T. OIKhBERG And E. RicARD, Operator spaces with few completely bounded maps, Math. Ann. 328 (2004), 229-259.

22. T. OikhBerg And H. Rosenthal, Extension properties for the space of compact operators, J. Funct. Analysis 79 (2001), 251-308.

23. G. PISIER, An introduction to the theory of operator spaces (Cambridge University Press, 2003).

24. A.-M. Popa And Z.-J. RuAn, Uniformly complete quotient space $U C Q(G)$ and the completely isometric representation of $U C Q(G)^{*}$ on $B\left(L_{2}(G)\right)$, Proc. Am. Math. Soc. 134 (2006), 1223-1235.

25. R. SMith AND N. SpRonk, Representations of group algebras in spaces of completely bounded maps, Indiana Univ. Math. J. 54 (2005), 873-896.

26. E. Størmer, Regular abelian Banach algebras of linear maps of operator algebras, $J$. Funct. Analysis 37 (1980), 331-373.

27. J. TAYLOR, Topological invariants of the maximal ideal space of a Banach algebra, Adv. Math. 19 (1976), 149-206.

28. A. ZsÁk, A Banach space whose operator algebra has $K_{0}$-group $\mathbb{Q}$, Proc. Lond. Math. Soc. 84 (2002), 747-768. 